\title{
A model of twenty-three metabolic -related genes predicting overall survival for lung adenocarcinoma
}

\author{
Zhenyu Zhao ${ }^{1,2}$, Boxue He ${ }^{1,2}$, Qidong Cai ${ }^{1,2}$, Pengfei Zhang ${ }^{1,2}$, Xiong Peng ${ }^{1,2}$, Yuqian Zhang ${ }^{1,2}$, Hui Xie ${ }^{1,2}$, Xiang $^{2}$ \\ Wang Corresp. 1,2 \\ 1 Department of Thoracic Surgery, The Second Xiangya Hospital of Central South University, 410011, Central South University, Changsha, Hunan, China \\ 2 Hunan Key Laboratory of Early Diagnosis and Precise Treatment of Lung Cancer, The Second Xiangya Hospital of Central South University, 410011 , \\ Central South University, Changsha, Hunan, China
}

Corresponding Author: Xiang Wang

Email address: wangxiang@csu.edu.cn

Background: The highest rate of cancer-related deaths worldwide is from lung adenocarcinoma (LUAD) annually. Metabolism was associated with tumorigenesis and cancer development. Metabolic-related genes may be important biomarkers and metabolic therapeutic targets for LUAD

Materials and Methods: In this study, the gleaned cohort included LUAD RNA-SEQ data from the Cancer Genome Atlas (TCGA) and corresponding clinical data $(n=445)$. The training cohort was utilized to model construction, and data from the Gene Expression Omnibus (GEO, GSE30219 cohort, $n=83$; GEO, GSE72094, $n=393$ ) were regarded as a testing cohort and utilized for validation. First, we used a lasso-penalized cox regression analysis to build a new metabolic-related signature for predicting the prognosis of LUAD patients. Next, we verified the metabolic gene model by survival analysis, C-index, receiver operating characteristic (ROC) analysis. Univariate and multivariate cox regression analyses were utilized to verify the gene signature as an independent prognostic factor. Finally, we constructed a nomogram and performed gene set enrichment analysis to facilitate subsequent clinical applications and molecular mechanism analysis.

Result: Patients with higher risk scores showed significantly associated with poorer survival. We also verified the signature can work as an independent prognostic factor for LUAD survival. The nomogram showed better clinical application performance for LUAD patient prognostic prediction. Finally, KEGG and GO pathways enrichment analyses suggested several especially enriched pathways, which may be helpful for us investigative the underlying mechanisms. 
1 Title: A model of twenty-three metabolic-related genes predicting overall

2 survival for lung adenocarcinoma

3 Author list:

4 Zhenyu Zhao ${ }^{1,2}$, Boxue He ${ }^{1,2}$, Qidong Cai ${ }^{1,2}$, Pengfei Zhang ${ }^{1,2}$, Xiong Peng ${ }^{1,2}$, Yuqian Zhang ${ }^{1,2}$, Hui Xie ${ }^{1,2}$,

5 Xiang Wang ${ }^{1,2}$

6 Affiliations:

$7 \quad{ }^{1}$ Department of Thoracic Surgery, The Second Xiangya Hospital of Central South University,

8410011 Changsha, Hunan, China

$9 \quad{ }^{2}$ Hunan Key Laboratory of Early Diagnosis and Precise Treatment of Lung Cancer, The Second

10 Xiangya Hospital of Central South University, 410011 Changsha, Hunan, China

12 Corresponding Author:

13 Xiang Wang ${ }^{1,2}$, M.D.

14 Department of Thoracic Surgery, The Second Xiangya Hospital of Central South 24 University.

15 Address:139 Renmin Road, Changsha/Hunan Province, 410011, China

16 Tel: +86 15874978585

17 Email address: email: wangxiang@.csu.edu.cn

\section{Abstract}

Background: The highest rate of cancer-related deaths worldwide is from lung adenocarcinoma (LUAD) annually. Metabolism was associated with tumorigenesis and cancer development. Metabolic-related genes may be important biomarkers and metabolic therapeutic targets for LUAD

Materials and Methods: In this study, the gleaned cohort included LUAD RNA-SEQ data from the Cancer Genome Atlas (TCGA) and corresponding clinical data $(n=445)$. The training cohort was utilized to model construction, and data from the Gene Expression Omnibus (GEO, GSE30219 cohort, $\mathrm{n}=83$; GEO, GSE72094, $\mathrm{n}=393$ ) were regarded as a testing cohort and utilized for validation. First, we used a lasso-penalized cox regression analysis to build a new metabolic-related signature for predicting the prognosis of LUAD patients. Next, we verified the metabolic gene model by survival analysis, C-index, receiver operating characteristic (ROC) 
31 analysis. Univariate and multivariate cox regression analyses were utilized to verify the gene

32 signature as an independent prognostic factor. Finally, we constructed a nomogram and

33 performed gene set enrichment analysis to facilitate subsequent clinical applications and

34 molecular mechanism analysis.

35 Result: Patients with higher risk scores showed significantly associated with poorer survival. We

36 also verified the signature can work as an independent prognostic factor for LUAD survival. The

37 nomogram showed better clinical application performance for LUAD patient prognostic

38 prediction. Finally, KEGG and GO pathways enrichment analyses suggested several especially

39 enriched pathways, which may be helpful for us investigative the underlying mechanisms.

Keywords: Metabolic, prognostic, signature, lung adenocarcinoma

\section{Introduction}

Lung cancer (LC) is one of the most common cancers worldwide and the main cause of cancerrelated mortality (Bray et al.,2018; Torre et al.,2015). Non-small cell lung cancer (NSCLC) accounts for $85 \%$ of all LCs. The 5-year survival rate after the diagnosis of LC is $15.6 \%$ (Nanavaty Alvarez \& Alberts,2014). In NSCLC, lung adenocarcinoma (LUAD) is the major histological subtype (Balzer et al.,2018), and the recurrence rate and mortality rate remain high despite recent advances in surgical methods, neoadjuvant therapies, and immunotherapies.

As bioinformatics advances in oncology research, researchers can utilize access public resources from multiple public databases such as The Cancer Genome Atlas (TCGA) and the Gene Expression Omnibus (GEO), as well as Surveillance and Epidemiology and End Results (SEER). (Doll Rademaker \& Sosa,2018; Li et al.,2018; Liu et al.,2019). Bioinformatics has contributed to determining the prognosis and treatment of LC (Parikh,2019). There have been numerous studies on gene prognosis models that could contribute to the selection of LC treatment methods and the prediction of survival after LC surgery; for example, a prognostic signature containing 6 genes (RRAGB, RSPH9, RPS6KL1, RXFP1, RRM2, and RTL) to evaluate the prognosis of NSCLC patients (Xie \& Xie,2019). In another article on prognostic characteristics of LUAD, a prognostic model based on 20 genes was developed to predict patient overall survival (OS) (Zhao Li \& Tian,2018). These prognostic signatures all have better clinical application performance.

Metabolic changes in LC are the key to diagnosis, and metabolic remodelling is a critical factor in tumorigenesis and development (Chen et al.,2019b). Metabolic remodelling not only provides substances and energy for the survival and proliferation of tumour cells but also protects tumour cells so that they can survive, proliferate, and metastasize in harsh microenvironments (Hensley et al.,2016). Therefore, changes in metabolism affect tumour prognosis and treatment effects (Chang Fang \& Gu,2020; Cruz-Bermúdez et al.,2019). To explore the correlation between metabolic genes and the prognosis of LUAD patients, we utilized the TCGA-LUAD database to 
70 build a prognostic signature of multiple metabolic-related genes and validated it in GEO data sets

71 for LUAD patients. We conducted this study, and our findings suggested that metabolic-related

72 gene signatures may be a prognostic marker for LUAD patients

73

\section{MATERIALS AND METHODS}

\subsection{Data collections}

First, we got clinical information for patients with LUAD from TCGA (https://portal.gdc.cancer.gov/). It included 497 LUAD patients with mRNA expression profiles and clinical follow up information was available for our study. The number of obtainable clinical cases for the selected subjects was 445 after removing 52 patient samples from the study due to a lack of clinical information (such as survival time, $\mathrm{T}$ stage, $\mathrm{N}$ stage, and so on) or survival time less than 30 days (avoiding non-cancer-related death samples). A total of 445 LUAD patients and their information were utilized to build a training cohort for identifying prognostic metabolic-related genes and building a prognostic risk model. Next, we downloaded the LUAD gene expression data from GEO (https://www.ncbi.nlm.nih.gov/geo/) in two accessed datasets GSE30219 and GSE72094. Removing other cancer pathological types, such as lung squamous cell carcinoma, 83 LUAD samples, and 393 LUAD samples were utilized to build a testing cohort for validating the prognostic value of the TCGA-LUAD prognostic risk model (up to April 01, 2020).

\subsection{Identification of metabolic-related genes in TCGA-LUAD}

First, we obtained 944 hub metabolism-related genes from the intersection of the MSigDB database (https://www.gsea-msigdb.org/gsea/msigdb) and TCGA-LUAD. Then, a Wilcoxon signed-rank test was performed on normal and cancer tissues in the training cohort by "limma" $\mathrm{R}$ package ( $|\log \mathrm{FC}|>0.5$; FC: fold change; a false discovery rate (FDR) $P<0.05$ ) (Diboun et al.,2006). The heatmap was plotted by the "pheatmap" $R$ package and we obtained 336 metabolic-related differentially expressed genes. Second, after univariate cox regression analysis, 59 metabolic genes were retained $(P<0.05)$ by using the method that the correlation between expression values of metabolic genes and survival of samples in the training cohort. Last, we performed lasso-penalized cox regression analysis to identify more important metabolic genes for OS prediction through the "glmnet, survival" R package (Zhang et al.,2019). We obtained twenty-three metabolic-related genes for risk model building. The three-step screening method was robust and performed via Perl (https://www.perl.org/) and R (version 3.6.1).

\subsection{Building the prognostic metabolic gene signature}

To construct the prognostic model, we utilized lasso-penalized cox regression analysis to select the prognostic metabolic-related gene (Tibshirani,1997). We obtained a risk score for each patient by their coefficient. Risk score $=($ Coef AKR1A1 $\times$ expression of AKR1A1) $+($ Coef NT5E $\times$ expression of NT5E) $+\ldots \ldots$ (Coef TYMS $\times$ expression of TYMS) (Liu et al.,2020). R 
109 software packages "survival" and "survminer" were used to calculate the optimal cut-off value

110 for risk scores and plot Kaplan-Meier survival curves (Chan et al.,2018). Using the median as a

111 point of differentiation, we differentiated patients into two groups: high-risk and low-risk. The $\mathrm{R}$

112 package "survivalROC" was used to plot time-dependent ROC curves for predicting the

113 diagnostic value (Heagerty Lumley \& Pepe,2000). The concordance index (C-index) was used to

114 evaluate the predictive ability of the risk model.

115

$116 \quad 2.4$ Verification of the prognostic signature as an independent risk factor and correlation

117 analysis between the clinical characteristics and risk scores

118 Patients with complete information on the corresponding clinical data were available for

119 univariate and multivariate analysis. $P<0.05$ symbolizes statistically significant (Liu et

120 al.,2020). We performing the Student's t-test to verify the correlation between clinical

121 characteristics and risk scores.

122

123

2.5 Construction and verification of the predictive nomogram

124 The nomogram was built by the "rms" $\mathrm{R}$ package according to training cohort data (Iasonos et

125 al.,2008). In our study, the tumour-node metastasis (TNM) model and the prognostic signature

126 were integrated into the predictive nomogram. We performed the calibration plot and C-index to

127 investigate the predictive ability of the nomogram. The calibration plot was used to assess

128 whether the numerical value of the predicted value of the model and the probability of the

129 occurrence of the ending event were consistent (Fenlon et al.,2018). We used C-index to assess

130 the predictive ability of the nomogram. It estimates the probability that the predicted result is

131 consistent with the actual observed result. We compared the TNM model, prognostic model, and

132 the nomogram model through ROC analysis and C-index (Liu et al.,2020). Next, we verified the

133 nomogram by $\mathrm{C}$-index, ROC analysis, and calibration plot in the testing cohort data.

134

1352.6 KEGG and GO pathways enrichment analyses

136 To study the biological role of mRNA markers in LUAD patients, we utilized the Kyoto

137 Encyclopedia of Genes and Genomics (KEGG) and Gene Ontology (GO) pathway enrichment

138 analysis to explore which pathways the differentially expressed genes were mainly enriched in

139 (up to April 11, 2020). Gene Set Enrichment Analysis (GESA) (https://www.gsea-

140 msigdb.org/gsea/index.jsp) was utilized to find enriched terms in the training cohort or testing

141 cohort (Subramanian et al.,2005). We choose "c2. cp. kegg. v6.2. symbols. gmt. gene sets" as a

142 reference gene set from the MSigDB database. (https://www.gsea-msigdb.org/gsea/msigdb/). $P$

$143<0.05$, FDR q-value $<0.25$, and normalized enrichment score $|\mathrm{NES}| \geq 1$ suggested statistically

144 significant. We plotted the results by "ggplot2, gridExtra, grid, plyr" R package. All operations

145 are carried out in GSEA_4.0.3. GO pathway enrichment analysis of metabolic genes was

146 performed by “clusterProfiler, org.Hs.eg.db, plot, ggplot2" in R package (Pathan et al.,2015) 
148

149

150

151

152

153

154

155

156

157

158

159

160

161

162

163

164

165

166

167

168

169

170

171

172

173

174

175

176

177

178

179

180

181

182

183

184

185

\subsection{Statistical analyses}

$\log \mathrm{FC}$ : logarithmic value of FC; positive/negative $\log \mathrm{FC}$ indicates the logarithmic foldness of upregulation/downregulation; $|\log \mathrm{FC}|>0.5$ indicates multiple differences in the gene expression greater than 0.5 between normal tissues and cancer tissues. A coefficient is a number that expresses a measurement of a particular quality of a substance or object under specified conditions (Bøvelstad et al.,2007). The risk score was calculated according to the formula:

Risk score (patients $)=\sum_{n}(\operatorname{coefficient}(m R N A n) * \operatorname{expression}(m R N A n))$.

C-index had a lower accuracy from $0.50-0.70$, medium accuracy between $0.71-0.90$, and higher accuracy when greater than 0.90 degrees (Kim Schaubel \& McCullough,2018). The Area Under Curve (AUC) value of the time-dependent ROC lay in the range of $0.5-0.9$ viewed as statistically significant. All statistical analyses were conducted through R 3.6.1.

\section{RESULTS}

\subsection{Construction of the prognostic signature from the training cohort}

The workflow of the study was shown in Fig 1. In our study, 445 LUAD patients (Table S1, clinical information in Table S1) from the TCGA data set were assigned to the training sample cohort; 83 LUAD patients (Table S3, clinical information in Table S4) and 393 LUAD patients (Table S5, clinical information in Table S6) from the GEO data set were assigned to the testing sample cohort for batch processing. After the Wilcoxon signed-rank test was applied to the training set, we obtained 336 meaningful metabolic genes (Figure 2, Table S7). From the 336 meaningful metabolic genes, we got 59 mRNAs which were considered to be significantly associated with OS in LUAD patients, 42 high-risk genes, and 17 low-risk genes (Figure 3, Table S8). Finally, lasso-penalized Cox analysis identified 24 genes (AKR1A1, NT5E, PTGIS, GMPS, MBOAT1, ADCY9, B4GALT1, MAOB, INPP4B, NEU1, ALDOA, ENTPD2, GNPNAT1, GSTA3, INPP5A, PKM, HK3, ALDH2, AK2, LDHA, CHPT1, SMS, CTPS2, and TYMS) to construct the prognostic model (Table 1).

\subsection{The twenty-three metabolic genes signature and predictability assessment in the} training cohort

Finding that there is a significant and independent correlation between the expression of twentythree prognostic mRNAs and OS, we believed that combining the 23 metabolic genes to form a twenty-three gene signature could predict a patient's prognosis. According to the optimal cut-off of 1.53, we classified training cohort samples into two groups: a high-risk group $(\mathrm{n}=222)$ and a low-risk group $(\mathrm{n}=223)($ Table $S 9)$. The OS rate between the two risk groups was significantly different $(P=5.543 \mathrm{e}-10)$ (Figure $4 A)$.

The AUC was $0.798,0.747$, and 0.734 for the 1,3 , and 5 -year OS, separately (Figure $4 B$ ). We plotted patients' risk curves in the training cohort and analysed their distribution in Fig $4 D-E$, 
186 and the heat map reveals the prognostic mRNA expression patterns between two distinct

187 prognostic patient groups (Figure 4C).

188

\subsection{Validation of the twenty-three metabolic gene signatures}

To test the robustness of the prognostic signature, according to the risk score cut-off of the training cohort, GSE30219 was structured into a high-risk group $(\mathrm{n}=25)$ and a low-risk group $(\mathrm{n}$ = 58) (Table S10); and GSE72094 was structured into a high-risk group $(\mathrm{n}=196)$ and a low-risk group $(\mathrm{n}=197)$ (Table S11). In the GSE30219 cohort, the Kaplan-Meier survival curves of the prognostic signature have a statistically significant difference in the two predicted risk groups (Figure 5, $P=1.176 \mathrm{e}-02$ ). The AUC was $0.694,0.645$, and 0.637 for the 1,3 , and 5-year OS, separately (Figure 5B). The risk curves and expression of twenty-three metabolic genes in the GSE30219 cohort were shown in Fig 5C-E. And in the GSE72094 cohort, Kaplan-Meier survival curves of the prognostic signature has a statistically significant difference in the two predicted risk groups (Figure 6, $P=1.417 \mathrm{e}-10$ ). The AUC was $0.695,0.725$, and 0.742 for the 1 , 3 , and 5-year OS, separately (Figure 6B). The risk curves and expression of the twenty-three metabolic genes in the GSE30219 cohort were shown in Fig 6C-E. We also compared our results with the two published gene signature studies (Xie \& Xie,2019; Zhao Li \& Tian,2018) and found that the $\mathrm{C}$-index results were better than their signatures (Table 2).

204

Taken together, these results indicated a better predictive performance about our prognostic

206

207 signature.

\subsection{Correlation analysis between the prognostic signature and clinical characteristics} 445 patients with their information from TCGA-LUAD cohort were utilized for the correlation analysis. Being male $(P=0.017)$ and TNM stage $(P<0.01)$ have a significant correlation with a higher risk score. Samples with higher T, N, and M grading were also significantly correlated with a higher risk score. (Table 3).

213

83 patients with their information from GSE30219 cohort were utilized for the correlation analysis. TNM stage was significantly associated with a higher risk score $(P<0.001)$. Samples with higher $\mathrm{T}, \mathrm{N}$, and $\mathrm{M}$ grading were also significantly correlated with a higher risk score $(P<$ 0.01) (Table 3). And 393 patients with their information from GSE72094 cohort were utilized for the correlation analysis. TNM stage was significantly correlated with a higher risk score $(P<$

220 0.001) (Table 3).

\subsection{Validation of the independent prognostic factor}

We analysed 445 patients, with a median age of 68 , grouping them by gender (Table 4 ). The results identified that our prognostic signature was an independent OS prognostic indicator 
225

226

227

228

229

230

231

232

233

234

235

236

237

238

239

240

241

242

243

244

245

246

247

248

249

250

251

252

253

254

255

256

257

258

259

260

261

262

263

prognostic indicator in the GSE30219 cohort (Figure 7C and 7D) and GSE72094 cohort (Figure $7 E$ and $7 F$ ). The multivariate Cox analysis indicating that the prognostic signature was

significantly associated with OS in each cohort when adjusted for the TNM stage (Table 4). And stratification analysis showed that high-risk group was significantly correlated with a poorer OS (Figure 8A-F). However, in the GSE30219 testing cohort, patients in the high-risk group from TNM stages III and IV show no significantly correlated with OS (Figure $8 D$ ).

\subsection{Construction and verification of the predictive nomogram}

we built the nomogram by including the independent prognostic roles (Figure 9A). Calibration plots verified the performance of the nomogram (Figure 9B). The C-index of the TNM model, prognostic signature, and nomogram model were $0.654,0.730$, and 0.793 , separately (Table 5). The AUC was $0.838,0.785$, and 0.779 for the 1,3 , and 5-year OS, respectively. The nomogram model showed a better AUC predicting 1, 3, and 5-year OS than the prognostic model in the training cohort (Figure 9C-E).

Next, we verified the clinical application of the nomogram in the GSE30219 and GSE72094 cohorts. In the GSE30219 and GSE72094 testing cohorts, calibration plots verified the performance of the nomogram (Figure 10A and 10E). In the GSE30219 testing cohort, the Cindex of the TNM model, prognostic signature, and nomogram model were $0.612,0.682$, and 0.684 (Table 5). The AUC was 0.731, 0686, and 0.722 for the 1, 3, and 5-year OS, separately (Figure 10B-D). In the GSE72094 testing cohort, the C-index of the TNM model, prognostic signature, and nomogram model were $0.579,0.709$, and 0.713 , separately (Table 5). The AUC was $0.708,0.794$, and 0.771 for the 1,3 , and 5-year OS, separately (Figure 10F-H). The nomogram model identified the better AUC predicting 1, 3, and 5-year OS than the prognostic signature in both testing cohorts.

Taken together, the nomogram model increased the predicting ability of the prognostic signature. These results indicated the better predictive performance of the nomogram model.

\subsection{KEGG and GO pathways enrichment analyses}

KEGG enrichment analyses suggesting that a majority of the metabolism-related pathways such as the metabolism of fatty acid, arachidonic acid, glycerophospholipid, alpha-linolenic acid, and pyrimidine were associated with the low-risk group, while the cell cycle, mismatch repair, ubiquitin-mediated proteolysis, and p53 signalling pathways were associated with the high-risk group (Figure 11A, Table 6, Table S12). Besides, we performed GO pathway enrichment analysis on 23 genes. These 23 genes were statistically significant in metabolic-related pathways, such as nucleotide biosynthetic processes, secretory granule lumen, and monosaccharide binding in Biological Processes (BP), cellular component (CC), and molecular function (MF). (Figure 11B, Table S13).

Peer] reviewing PDF | (2020:05:48908:2:0:NEW 25 Aug 2020) 


\section{DISCUSSION}

266

267

268

269

270

271

272

273

274

275

276

277

278

279

280

281

282

283

284

285

286

287

288

289

290

291

292

293

294

295

296

297

298

299

300

301

302

Lung cancer has the highest mortality rate among cancer-related diseases worldwide (Bray et al.,2018). LUAD is the principal type of LC, with a percentage of more than half of morbidity and mortality in this group of patients (Jemal et al.,2017). With the increasing exploration of cancer metabolic heterogeneity, metabolic genes can work as a prognostic signature for LUAD. Identification of metabolism-related gene preferences and dependence mechanisms in tumour regulation has become increasingly important (Peng et al.,2017). Metabolic changes in LC are strategic to the diagnosis and influence the prognosis and response to treatment (Cruz-Bermúdez et al.,2019). TCGA and GEO databases already have a large amount of RNA-seq data from tumour samples in multiple cancers. Prognostic signatures of LUAD have been built and developed utilizing the public databases (Shang et al.,2017). Several metabolic genes for LUAD, such as TKT, ALDOA, TSC1, and CYP2A6, have been demonstrated to be related to the OS of LUAD (Lin et al.,2011; Wassenaar et al.,2015). Therefore, we investigated the relationship between tumour metabolism-related genes and the prognosis of LUAD. We first built twentythree metabolic-related gene prognostic signatures, which may be helpful for the diagnosis, treatment, and prognosis of LUAD.

In this study, we identified an efficient twenty-three metabolic-related gene prognostic model based on the TCGA-LUAD databases. Our prognostic model had better performance in predicting patients' prognosis. We also verified the performance of the prognostic signature in the testing cohort, and the results confirm the prognostic value of the prognostic signature. It is worth noting that in the GSE30219 databases, the high-risk group did not show a significant correlation with poorer survival in TNM stages III and IV when compared with the low-risk group. One possible reason is that with improvements in diagnostic technology, more and more early patients are diagnosed at TNM stages I and II, and fewer patients are diagnosed at TNM stages III and IV. The AUC of the ROC analysis from the TCGA-LUAD cohort and GEO cohorts verified the performance of the prognostic signature. Besides, the nomogram model showed better performance in prognosis predicting. We think that the nomogram will help us to make the clinical treatment strategy in the future. In conclusion, these results demonstrated a significant prognostic value of our prognostic model.

To better understand the molecular mechanism of metabolic genes, we enriched ten KEGG signalling pathways that are significantly related to metabolic gene models through GSEA. We found that patients in low-risk groups may profit from metabolic therapies. whereas, the outcomes provide feasible guidance for explaining the unknown mechanisms of labelling. GO analysis also indicated the twenty-three genes that were enriched in metabolic pathways. In conclusion, our signatures may reflect the disorder of LUAD patient tumour microenvironment and provide molecule biomarkers for treatment and prediction of the prognosis of LUAD. 
304 In our twenty-three gene prognosis model, several metabolic genes, including GSTA3, ENTPD2,

305

306

307

308

309

310

311

312

313

314

315

316

317

318

319

320

321

322

323

324

325

326

327

328

329

330

331

332

333

334

335

336

337

338

339

340

341 HK3, CHPT1, CTPS2, and ADCY9, were confirmed for the first time to be correlated with the prognosis of LUAD. GSTA3 is recognized as an antioxidative protease (Chen et al.,2019a). Studies of genetic analysis models and the mechanism of GSTA3 found that it was associated with tumour prognosis (Bruzzoni-Giovanelli et al.,2015). For example, GSTA3 was identified and regarded as a prognostic biomarker for nasopharyngeal carcinoma and gastric cancer (Duan et al.,2018; Zhang Wu \& Cheng,2019). GSTA3 inhibits HSC activation and liver fibrosis by inhibiting MAPK and GSK-3 $\beta$ signalling pathways, suggesting that GSTA3 could be a feasible target for therapeutic interventions for liver fibrosis (Chen et al.,2019a). GSTA3 overexpression in breast cancer cells stimulates proliferation and inhibits apoptosis, which leads to chemotherapy resistance and radiation resistance in tumour cells (Thewes et al.,2010).

Overexpression of ENTPD2 can be regarded as a poor prognostic indicator of liver cancer. In an anoxic environment, ENTPD2 converts extracellular ATP into 5'-AMP, which inhibits the differentiation of myeloid inhibitory cells (MDSC) and promotes the stability of MDSC. The inhibition of ENTPD2 expression can reduce cancer growth and improve the effectiveness of immune checkpoint inhibitors (Chiu et al.,2017). HK3 is a very well-known glycolysis gene whose overexpression could be linked to hypoxia-induced upregulation of glycolysis and improvement in breast cancer cell survival (Jarrar et al.,2020). The pre-expression of HK3 is related to the epithelial-mesenchymal transition in colorectal cancer (CRC) and maybe a strategic metabolic gene for rapid proliferation, survival, and metastasis of CRC cells (Pudova et al.,2018). Besides, HK3 is associated with a CpG island methylated phenotype (CIMP) in colon adenocarcinoma (COAD). The upregulation of HK3 was reported in CIMP-high tumours compared to non-CIMP ones. HK3 can serve as a biomarker of high CIMP status in COAD (Fedorova et al.,2019). CHPT1 is considered to be a direct oestrogen receptor $\alpha$-regulatory gene and is necessary for oestrogen-induced choline metabolism. CHPT1 mediates metabolic changes in breast cancer cells, and silencing CHPT1 can inhibit breast cancer cell proliferation and early metastasis of tamoxifen-resistant breast cancer cells, suggesting that CHPT1 is a treatment target for cancers (Fedorova et al.,2019; Jia et al.,2016). CTPS2 had a profound effect on osteosarcoma metastasis (Fan et al.,2019) and also participated in the primary immunodeficiency of herpes virus susceptible populations (Verzegnassi et al.,2018). Low CTPS2 expression may be the underlying determinant of 5FU resistance (Tan et al.,2011). ADCY9 regulates signalling pathways mainly by producing a second messenger cyclic adenosine monophosphate. Some research found that ADCY9 acts as a key gene in the cisplatin response regulatory network in the pro-apoptotic stage in breast cancer treatment (Fallahi \& Godini,2019), and overexpression of ADCY9 is a poor prognostic marker for disease-free survival in colon cancer (Yi et al.,2018). The above studies provided us with directions for studying these six genes in LUAD.

In addition to the above genes, many metabolic-related genes that were linked to the prognosis of 
342 the LUAD were included in seventeen additional genes, such as ALDH2, PKM, LDHA, and

343 SMS. First, some of them, such as INPP4B, ALDOA, ALDH2, and LDHA, are significantly

344 involved in LUAD suppression. INPP4B is regarded as a tumour suppressor of LC because it

345 regulates the level of 3-phosphorylated phosphoinositide at the cellular level and activates

346 phosphoinositide in PTEN-deficient cells (Vo \& Fruman,2015). ALDOA is a critical enzyme

347 involved in metabolic reprogramming and metastasis of NSCLC that increases the ability of LC

348 cells to migrate and invade by interacting with $\gamma$-actin. Blocking this interaction could be an

349 effective cancer treatment (Chang et al.,2019). The expression level of ALDH2 was substantially

350 correlated with a poor prognosis in LUAD (Chen et al.,2018). The principal role of ALDH2 is

351 detoxifying acetaldehyde (ACE) to non-toxic acetic acid. ALDH2 inhibition leads to the

352 accumulation of ACE, which enhances the migration of LUAD cells by damaging DNA.

353 Therefore, activating ALDH2 could provide a novel strategy for treating LUAD (Li et al.,2019).

354 LDHA is an essential enzyme for glucose metabolism. It can inhibit the expression of HIF-1 $\alpha$

355 and its downstream gene GLUT1 and thus inhibit the growth of NSCLC cells (Massari et

356 al.,2016). Some additional genes are associate with tumour treatment tolerance. For example,

357 low expression of MOBA inhibits the NF- $\kappa B$ signalling pathway, leading to NSCLC

358 radioresistance (Son et al.,2016). In another study, silencing TYMS increased the sensitivity of

359 NSCLC tumour cells to pemetrexed (Agulló-Ortuño et al.,2020). In addition, several metabolic

360 genes have also been confirmed to be closely related to the occurrence of LC and have provided

361 some novel research directions. For instance, B4GALT1 is related to aberrant gene promoter

362 methylation and maintains the stemness of LC stem cells (Zhang Zhang \& Yu,2019). SMS

363 participates in the lymphatic metastasis of LUAD (Lemay et al.,2019).

364

365 Compared to other studies, we first used metabolic-related genes to build a prognosis model

366 from TCGA-LUAD and validated it in two GEO datasets. Our gene signature also had a better

367

368 prediction ability compared to the other model. This risk model perhaps provides potential biomarkers for studying the relationship of metabolic microenvironmental diseases, metabolic

370 therapies, and therapeutic responses. The risk model and TNM model were used to build the nomogram, and the calibration plot, C-index, ROC analysis validated the clinical application of the twenty-three metabolic-related gene signature, which may be helpful for the diagnosis, treatment, and prognosis of LUAD.

373

374

However, there are still some limitations in our study: First, the connection between 23

375 metabolic gene markers and the metabolic microenvironment needs to be verified by basic experiments. Secondly, a large number of clinical samples are lacking to verify whether the prognostic effect of metabolic therapy is related to its metabolic microenvironment. Basic and clinical trials will need to continue in the future to explore this relationship. Besides, some prognostic metabolic genes may not meet the screening criteria and were not included when 
381 of LUAD. Based on the above factors, the application of risk score to the clinic remains a huge

382 challenge

383

384

\section{Conclusion}

385

In conclusion, our research identified a 23 metabolic-related gene signature for LUAD patient

386 prognosis based on the TCGA data set. Our signature provides potential biomarkers for studying aspects of metabolic microenvironmental diseases, metabolic therapies, and therapeutic responses. However, it is still urgent to further investigate the relationship between metabolic microenvironment and metabolic therapy, and more functional experiments are required for revealing the mechanism of metabolic genes in the process of LUAD development.

391

\section{Acknowledgements}

393

The authors thank Professor Yongguang Tao for his comments and suggestions throughout the writing process. It is with regret that not all relevant studies could be cited due to space limitations.

\section{References}

Agulló-Ortuño M. T., García-Ruiz I., Díaz-García C. V., Enguita A. B., Pardo-Marqués V., Prieto-García E., Ponce S., Iglesias L., Zugazagoitia J., López-Martín J. A., Paz-Ares L., and Nuñez J. A. 2020. Blood mRNA expression of REV3L and TYMS as potential predictive biomarkers from platinum-based chemotherapy plus pemetrexed in non-small cell lung cancer patients. Journal 85:525-535. DOI: 10.1007/s00280-019-04008-9

Balzer B. W. R., Loo C., Lewis C. R., Trahair T. N., and Anazodo A. C. 2018. Adenocarcinoma of the Lung in Childhood and Adolescence: A Systematic Review. Journal 13:1832-1841. DOI: 10.1016/j.jtho.2018.08.2020

Bøvelstad H. M., Nygård S., Størvold H. L., Aldrin M., Borgan Ø, Frigessi A., and Lingjaerde O. C. 2007. Predicting survival from microarray data--a comparative study. Journal 23:2080-2087. DOI: 10.1093/bioinformatics/btm305

Bray F., Ferlay J., Soerjomataram I., Siegel R. L., Torre L. A., and Jemal A. 2018. Global cancer statistics 2018: GLOBOCAN estimates of incidence and mortality worldwide for 36 cancers in 185 countries. Journal 68:394-424. DOI: $10.3322 /$ caac.21492

Bruzzoni-Giovanelli H., González J. R., Sigaux F., Villoutreix B. O., Cayuela J. M., Guilhot J., Preudhomme C., Guilhot F., Poyet J. L., and Rousselot P. 2015. Genetic polymorphisms associated with increased risk of developing chronic myelogenous leukemia. Journal 6:36269-36277. DOI: 10.18632/oncotarget.5915

Chan A. W. H., Zhong J., Berhane S., Toyoda H., Cucchetti A., Shi K., Tada T., Chong C. C. N., Xiang B. D., Li L. Q., Lai P. B. S., Mazzaferro V., García-Fiñana M., Kudo M., Kumada T., Roayaie S., and Johnson P. J. 2018. Development of pre and post-operative models to predict early recurrence of hepatocellular carcinoma after surgical resection. Journal 69:1284-1293. DOI: 10.1016/j.jhep.2018.08.027

Chang L., Fang S., and Gu W. 2020. The Molecular Mechanism of Metabolic Remodeling in Lung Cancer. Journal 11:1403-1411. DOI: 10.7150/jca.31406

Chang Y. C., Chiou J., Yang Y. F., Su C. Y., Lin Y. F., Yang C. N., Lu P. J., Huang M. S., Yang C. J., and Hsiao M. 
420

421

422

423

424

425

426

427

428

429

430

431

432

433

434

435

436

437

438

439

440

441

442

443

444

445

446

447

448

449

450

451

452

453

454

455

456

457

458

2019. Therapeutic Targeting of Aldolase A Interactions Inhibits Lung Cancer Metastasis and Prolongs Survival. Journal 79:4754-4766. DOI: 10.1158/0008-5472.can-18-4080

Chen H., Gan Q., Yang C., Peng X., Qin J., Qiu S., Jiang Y., Tu S., He Y., Li S., Yang H., Tao L., and Peng Y. 2019a. A novel role of glutathione S-transferase A3 in inhibiting hepatic stellate cell activation and rat hepatic fibrosis. Journal 17:280. DOI: 10.1186/s12967-019-2027-8

Chen P. H., Cai L., Huffman K., Yang C., Kim J., Faubert B., Boroughs L., Ko B., Sudderth J., Mcmillan E. A., Girard L., Chen D., Peyton M., Shields M. D., Yao B., Shames D. S., Kim H. S., Timmons B., Sekine I., Britt R., Weber S., Byers L. A., Heymach J. V., Chen J., White M. A., Minna J. D., Xiao G., and Deberardinis R. J. 2019b. Metabolic Diversity in Human Non-Small Cell Lung Cancer Cells. Journal 76:838-851.e835. DOI: 10.1016/j.molcel.2019.08.028

Chen X., Legrand A. J., Cunniffe S., Hume S., Poletto M., Vaz B., Ramadan K., Yao D., and Dianov G. L. 2018. Interplay between base excision repair protein $\mathrm{XRCC} 1$ and $\mathrm{ALDH} 2$ predicts overall survival in lung and liver cancer patients. Journal 41:527-539. DOI: 10.1007/s13402-018-0390-8

Chiu D. K., Tse A. P., Xu I. M., Di Cui J., Lai R. K., Li L. L., Koh H. Y., Tsang F. H., Wei L. L., Wong C. M., Ng I. O., and Wong C. C. 2017. Hypoxia inducible factor HIF-1 promotes myeloid-derived suppressor cells accumulation through ENTPD2/CD39L1 in hepatocellular carcinoma. Journal 8:517. DOI: 10.1038/s41467017-00530-7

Cruz-Bermúdez A., Laza-Briviesca R., Vicente-Blanco R. J., García-Grande A., Coronado M. J., Laine-Menéndez S., Alfaro C., Sanchez J. C., Franco F., Calvo V., Romero A., Martin-Acosta P., Salas C., Garcia J. M., and Provencio M. 2019. Cancer-associated fibroblasts modify lung cancer metabolism involving ROS and TGF- $\beta$ signaling. Journal 130:163-173. DOI: 10.1016/j.freeradbiomed.2018.10.450

Diboun I., Wernisch L., Orengo C. A., and Koltzenburg M. 2006. Microarray analysis after RNA amplification can detect pronounced differences in gene expression using limma. Journal 7:252. DOI: 10.1186/1471-2164-7252

Doll K. M., Rademaker A., and Sosa J. A. 2018. Practical Guide to Surgical Data Sets: Surveillance, Epidemiology, and End Results (SEER) Database. Journal 153:588-589. DOI: 10.1001/jamasurg.2018.0501

Duan S., Gong B., Wang P., Huang H., Luo L., and Liu F. 2018. Novel prognostic biomarkers of gastric cancer based on gene expression microarray: COL12A1, GSTA3, FGA and FGG. Journal 18:3727-3736. DOI: 10.3892/mmr.2018.9368

Fallahi H., and Godini R. 2019. System-level responses to cisplatin in pro-apoptotic stages of breast cancer MCF-7 cell line. Journal 83:107155. DOI: 10.1016/j.compbiolchem.2019.107155

Fan H., Lu S., Wang S., and Zhang S. 2019. Identification of critical genes associated with human osteosarcoma metastasis based on integrated gene expression profiling. Journal 20:915-930. DOI: $10.3892 / \mathrm{mmr} .2019 .10323$

Fedorova M. S., Krasnov G. S., Lukyanova E. N., Zaretsky A. R., Dmitriev A. A., Melnikova N. V., Moskalev A. A., Kharitonov S. L., Pudova E. A., Guvatova Z. G., Kobelyatskaya A. A., Ishina I. A., Slavnova E. N., Lipatova A. V., Chernichenko M. A., Sidorov D. V., Popov A. Y., Kiseleva M. V., Kaprin A. D., Snezhkina A. V., and Kudryavtseva A. V. 2019. The CIMP-high phenotype is associated with energy metabolism alterations in colon adenocarcinoma. Journal 20:52. DOI: 10.1186/s12881-019-0771-5

Peer] reviewing PDF | (2020:05:48908:2:0:NEW 25 Aug 2020) 
459 Fenlon C., O'grady L., Doherty M. L., and Dunnion J. 2018. A discussion of calibration techniques for evaluating $460 \quad$ binary and categorical predictive models. Journal 149:107-114. DOI: 10.1016/j.prevetmed.2017.11.018

461 Heagerty P. J., Lumley T., and Pepe M. S. 2000. Time-dependent ROC curves for censored survival data and a diagnostic marker. Journal 56:337-344. DOI: DOI: 10.1111/j.0006-341x.2000.00337.x

463

464

465

466

467

468

469

470

471

472

473

474

475

476

477

478

479

480

481

482

483

484

485

486

487

488

489

490

491

492

493

494

495

496

497
Hensley C. T., Faubert B., Yuan Q., Lev-Cohain N., Jin E., Kim J., Jiang L., Ko B., Skelton R., Loudat L., Wodzak M., Klimko C., Mcmillan E., Butt Y., Ni M., Oliver D., Torrealba J., Malloy C. R., Kernstine K., Lenkinski R. E., and Deberardinis R. J. 2016. Metabolic Heterogeneity in Human Lung Tumors. Journal 164:681-694. DOI: $10.1016 /$ j.cell.2015.12.034

Iasonos A., Schrag D., Raj G. V., and Panageas K. S. 2008. How to build and interpret a nomogram for cancer prognosis. Journal 26:1364-1370. DOI: 10.1200/jco.2007.12.9791

Jarrar Y., Zihlif M., Al Bawab A. Q., and Sharab A. 2020. Effects of Intermittent Hypoxia on Expression of Glucose Metabolism Genes in MCF7 Breast Cancer Cell Line. Journal 20:216-222. DOI: $10.2174 / 1568009619666191116095847$

Jemal A., Ward E. M., Johnson C. J., Cronin K. A., Ma J., Ryerson B., Mariotto A., Lake A. J., Wilson R., Sherman R. L., Anderson R. N., Henley S. J., Kohler B. A., Penberthy L., Feuer E. J., and Weir H. K. 2017. Annual Report to the Nation on the Status of Cancer, 1975-2014, Featuring Survival. Journal 109. DOI: 10.1093/jnci/djx030

Jia M., Andreassen T., Jensen L., Bathen T. F., Sinha I., Gao H., Zhao C., Haldosen L. A., Cao Y., Girnita L., Moestue S. A., and Dahlman-Wright K. 2016. Estrogen Receptor $\alpha$ Promotes Breast Cancer by Reprogramming Choline Metabolism. Journal 76:5634-5646. DOI: 10.1158/0008-5472.can-15-2910

Kim S., Schaubel D. E., and Mccullough K. P. 2018. A C-index for recurrent event data: Application to hospitalizations among dialysis patients. Journal 74:734-743. DOI: 10.1111/biom.12761

Lemay A. M., Courtemanche O., Couttas T. A., Jamsari G., Gagné A., Bossé Y., Joubert P., Don A. S., and Marsolais D. 2019. High FA2H and UGT8 transcript levels predict hydroxylated hexosylceramide accumulation in lung adenocarcinoma. Journal 60:1776-1786. DOI: 10.1194/j1r.M093955

Li K., Guo W., Li Z., Wang Y., Sun B., Xu D., Ling J., Song H., Liao Y., Wang T., Jing B., Hu M., Kuang Y., Wang Q., Yao F., Sun A., Zhu L., Wang L., and Deng J. 2019. ALDH2 Repression Promotes Lung Tumor Progression via Accumulated Acetaldehyde and DNA Damage. Journal 21:602-614. DOI: 10.1016/j.neo.2019.03.008

Li Y., Gu J., Xu F., Zhu Q., Ge D., and Lu C. 2018. Transcriptomic and functional network features of lung squamous cell carcinoma through integrative analysis of GEO and TCGA data. Journal 8:15834. DOI: 10.1038/s41598018-34160-w

Lin C. C., Chen L. C., Tseng V. S., Yan J. J., Lai W. W., Su W. P., Lin C. H., Huang C. Y., and Su W. C. 2011. Malignant pleural effusion cells show aberrant glucose metabolism gene expression. Journal 37:1453-1465. DOI: $10.1183 / 09031936.00015710$

Liu G. M., Xie W. X., Zhang C. Y., and Xu J. W. 2020. Identification of a four-gene metabolic signature predicting overall survival for hepatocellular carcinoma. Journal 235:1624-1636. DOI: 10.1002/jcp.29081

Liu M., Zhou S., Wang J., Zhang Q., Yang S., Feng J., Xu B., and Zhong S. 2019. Identification of genes associated with survival of breast cancer patients. Journal 26:317-325. DOI: 10.1007/s12282-018-0926-9

Peer) reviewing PDF | (2020:05:48908:2:0:NEW 25 Aug 2020) 
Massari F., Ciccarese C., Santoni M., Iacovelli R., Mazzucchelli R., Piva F., Scarpelli M., Berardi R., Tortora G., Lopez-Beltran A., Cheng L., and Montironi R. 2016. Metabolic phenotype of bladder cancer. Journal 45:4657. DOI: $10.1016 /$ j.ctrv.2016.03.005

Nanavaty P., Alvarez M. S., and Alberts W. M. 2014. Lung cancer screening: advantages, controversies, and applications. Journal 21:9-14. DOI: 10.1177/107327481402100102

Parikh A. R. 2019. Lung Cancer Genomics. Journal 48:78-83. DOI: 10.5644/ama2006-124.244

Pathan M., Keerthikumar S., Ang C. S., Gangoda L., Quek C. Y., Williamson N. A., Mouradov D., Sieber O. M., Simpson R. J., Salim A., Bacic A., Hill A. F., Stroud D. A., Ryan M. T., Agbinya J. I., Mariadason J. M., Burgess A. W., and Mathivanan S. 2015. FunRich: An open access standalone functional enrichment and interaction network analysis tool. Journal 15:2597-2601. DOI: 10.1002/pmic.201400515

Peng F., Wang R., Zhang Y., Zhao Z., Zhou W., Chang Z., Liang H., Zhao W., Qi L., Guo Z., and Gu Y. 2017. Differential expression analysis at the individual level reveals a lncRNA prognostic signature for lung adenocarcinoma. Journal 16:98. DOI: 10.1186/s12943-017-0666-z

Pudova E. A., Kudryavtseva A. V., Fedorova M. S., Zaretsky A. R., Shcherbo D. S., Lukyanova E. N., Popov A. Y., Sadritdinova A. F., Abramov I. S., Kharitonov S. L., Krasnov G. S., Klimina K. M., Koroban N. V., Volchenko N. N., Nyushko K. M., Melnikova N. V., Chernichenko M. A., Sidorov D. V., Alekseev B. Y., Kiseleva M. V., Kaprin A. D., Dmitriev A. A., and Snezhkina A. V. 2018. HK3 overexpression associated with epithelial-mesenchymal transition in colorectal cancer. Journal 19:113. DOI: 10.1186/s12864-0184477-4

Shang J., Song Q., Yang Z., Li D., Chen W., Luo L., Wang Y., Yang J., and Li S. 2017. Identification of lung adenocarcinoma specific dysregulated genes with diagnostic and prognostic value across 27 TCGA cancer types. Journal 8:87292-87306. DOI: 10.18632/oncotarget.19823

Son B., Jun S. Y., Seo H., Youn H., Yang H. J., Kim W., Kim H. K., Kang C., and Youn B. 2016. Inhibitory effect of traditional oriental medicine-derived monoamine oxidase B inhibitor on radioresistance of non-small cell lung cancer. Journal 6:21986. DOI: 10.1038/srep21986

Subramanian A., Tamayo P., Mootha V. K., Mukherjee S., Ebert B. L., Gillette M. A., Paulovich A., Pomeroy S. L., Golub T. R., Lander E. S., and Mesirov J. P. 2005. Gene set enrichment analysis: a knowledge-based approach for interpreting genome-wide expression profiles. Journal 102:15545-15550. DOI: 10.1073/pnas.0506580102

Tan W. L., Bhattacharya B., Loh M., Balasubramanian I., Akram M., Dong D., Wong L., Thakkar B., Salto-Tellez M., Soo R. A., Fichtner I., Iacopetta B., and Soong R. 2011. Low cytosine triphosphate synthase 2 expression renders resistance to 5-fluorouracil in colorectal cancer. Journal 11:599-608. DOI: 10.4161/cbt.11.6.14670

Thewes V., Orso F., Jäger R., Eckert D., Schäfer S., Kirfel G., Garbe S., Taverna D., and Schorle H. 2010. Interference with activator protein-2 transcription factors leads to induction of apoptosis and an increase in chemo- and radiation-sensitivity in breast cancer cells. Journal 10:192. DOI: 10.1186/1471-2407-10-192

Tibshirani R. 1997. The lasso method for variable selection in the Cox model. Journal 16:385-395

Torre L. A., Bray F., Siegel R. L., Ferlay J., Lortet-Tieulent J., and Jemal A. 2015. Global cancer statistics, 2012. Journal 65:87-108. DOI: 10.3322/caac.21262

Verzegnassi F., Valencic E., Kiren V., Giurici N., Bianco A. M., Marcuzzi A., Vozzi D., Tommasini A., and Faletra

Peer] reviewing PDF | (2020:05:48908:2:0:NEW 25 Aug 2020) 
537

538

539

540

541

542

543

544

545

546

547

548

549

550

551

552

553

554

555

556

557

558

559
F. 2018. The Challenge of Next Generation Sequencing in a Boy With Severe Mononucleosis and EBVrelated Lymphoma. Journal 40:e323-e326. DOI: 10.1097/mph.0000000000001004

Vo T. T., and Fruman D. A. 2015. INPP4B Is a Tumor Suppressor in the Context of PTEN Deficiency. Journal 5:697700. DOI: 10.1158/2159-8290.cd-15-0609

Wassenaar C. A., Ye Y., Cai Q., Aldrich M. C., Knight J., Spitz M. R., Wu X., Blot W. J., and Tyndale R. F. 2015. CYP2A6 reduced activity gene variants confer reduction in lung cancer risk in African American smokers-findings from two independent populations. Journal 36:99-103. DOI: 10.1093/carcin/bgu235

Xie H., and Xie C. 2019. A Six-Gene Signature Predicts Survival of Adenocarcinoma Type of Non-Small-Cell Lung Cancer Patients: A Comprehensive Study Based on Integrated Analysis and Weighted Gene Coexpression Network. Journal 2019:4250613. DOI: 10.1155/2019/4250613

Yi H., Wang K., Jin J. F., Jin H., Yang L., Zou Y., Du B., and Liu X. 2018. Elevated Adenylyl Cyclase 9 Expression Is a Potential Prognostic Biomarker for Patients with Colon Cancer. Journal 24:19-25. DOI: $10.12659 / \mathrm{msm} .906002$

Zhang J. Z., Wu Z. H., and Cheng Q. 2019. Screening and identification of key biomarkers in nasopharyngeal carcinoma: Evidence from bioinformatic analysis. Journal 98:e17997. DOI: 10.1097/md.0000000000017997

Zhang L., Zhang Z., and Yu Z. 2019. Identification of a novel glycolysis-related gene signature for predicting metastasis and survival in patients with lung adenocarcinoma. Journal 17:423. DOI: 10.1186/s12967-01902173-2

Zhang M., Zhu K., Pu H., Wang Z., Zhao H., Zhang J., and Wang Y. 2019. An Immune-Related Signature Predicts Survival in Patients With Lung Adenocarcinoma. Journal 9:1314. DOI: 10.3389/fonc.2019.01314

Zhao K., Li Z., and Tian H. 2018. Twenty-gene-based prognostic model predicts lung adenocarcinoma survival. Journal 11:3415-3424. DOI: 10.2147/ott.s158638

Peer] reviewing PDF | (2020:05:48908:2:0:NEW 25 Aug 2020) 


\section{Figure 1}

\section{Workflow of the study.}

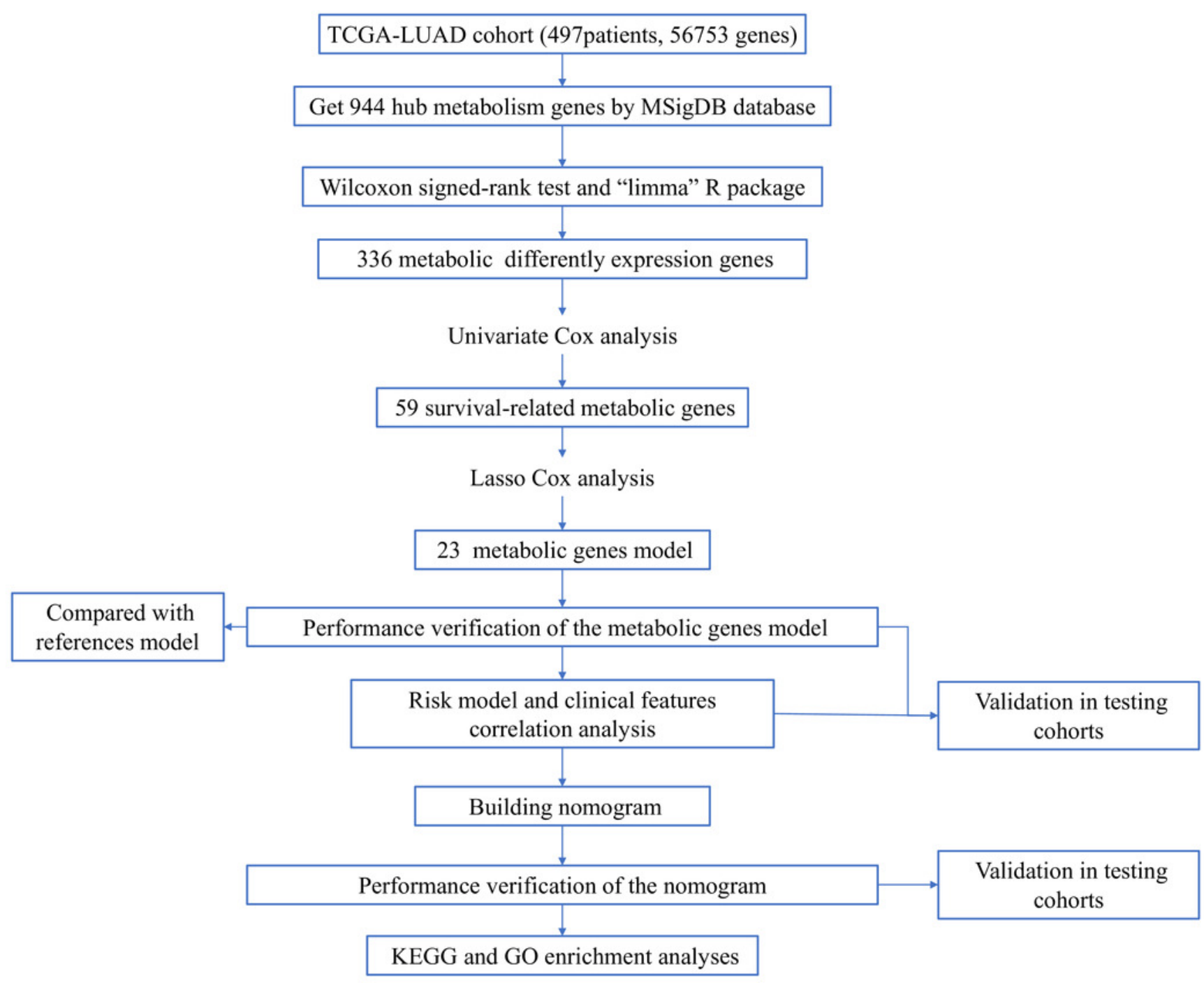




\section{Figure 2}

Heatmap and volcano map of the differentially expressed genes in normal and tumour tissues from TCGA-LUAD.

(A) Heatmap of the differentially expressed genes in normal and tumour tissues from TCGALUAD; (B) Volcano map of the differentially expressed genes in normal and tumour tissues from TCGA-LUAD. (In the volcano map, red: genes upregulated in tumour groups; yellow: genes downregulated in the tumour group; black: no differentially expressed genes in the tumour group.)

A

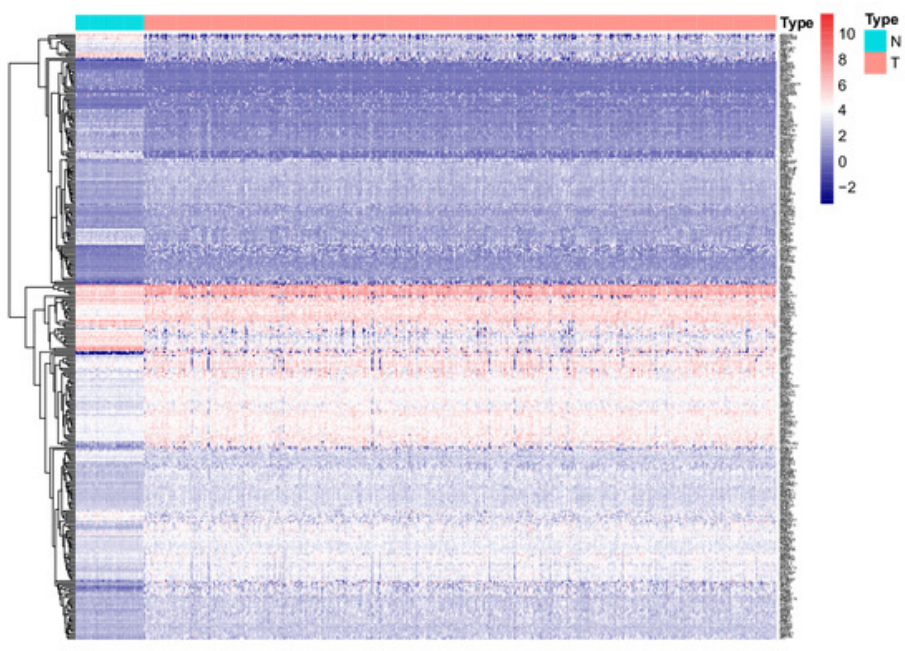

B

Volcano

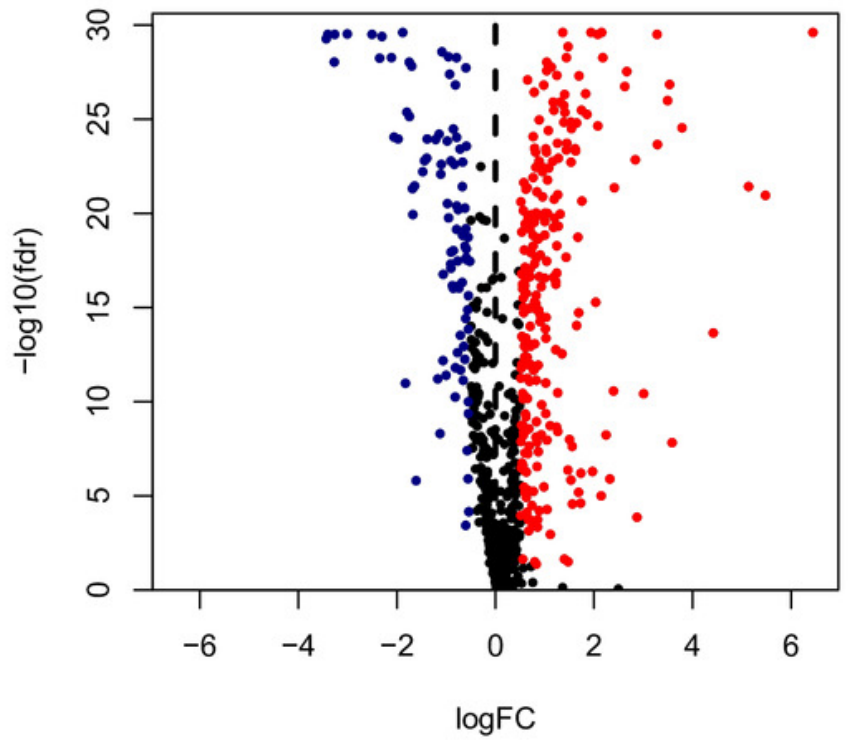


Figure 3

Forest map of the fifty-nine mRNAs (red: high-risk genes; yellow: low-risk genes).
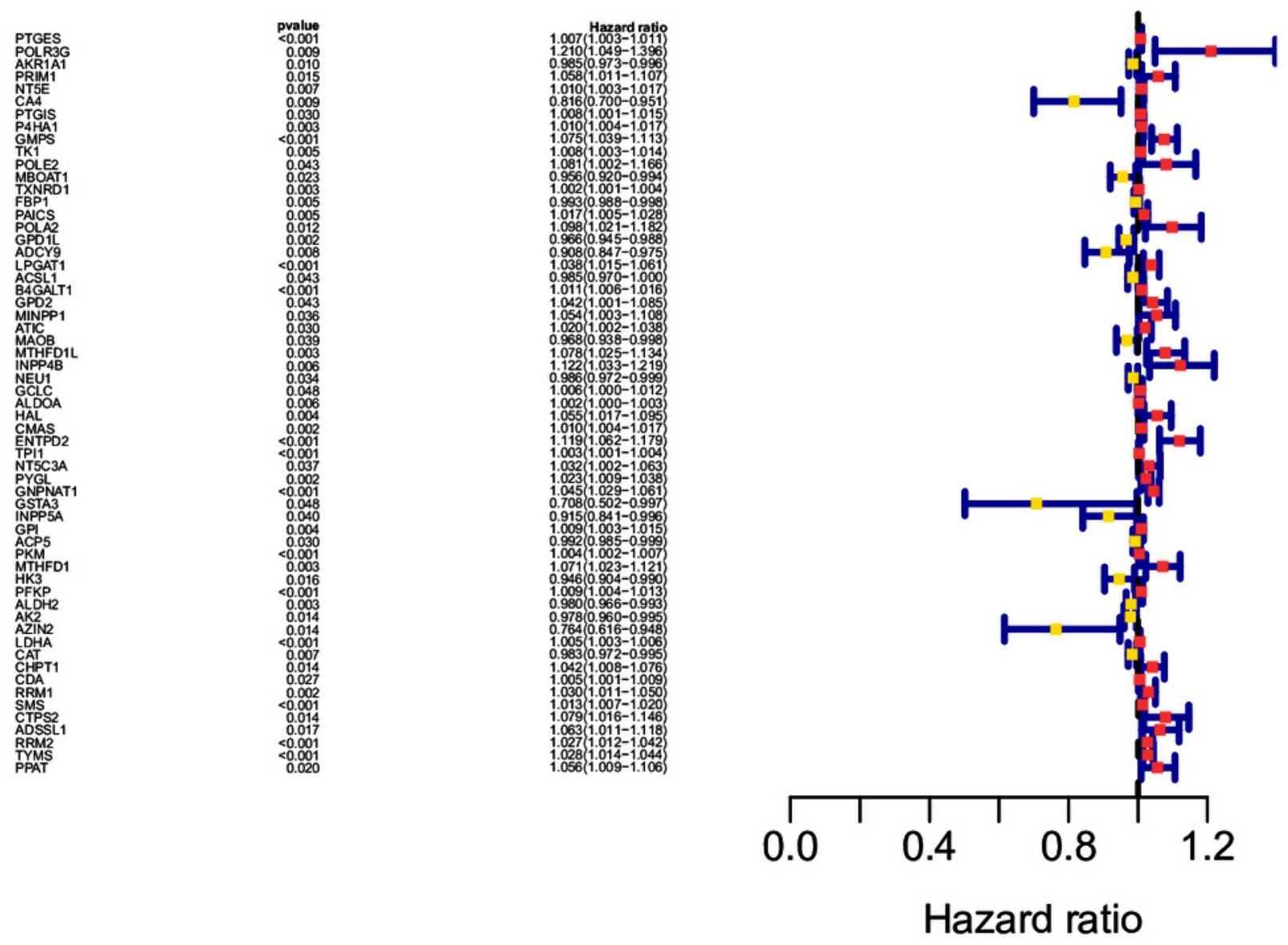
Figure 4

Kaplan-Meier analysis, time-dependent ROC analysis, and risk score analysis for the twenty-three gene signature in the TCGA-LUAD cohort.

(A) Kaplan-Meier curve of the twenty-three gene signature in the TCGA cohort; (B) Timedependent ROC analysis of the twenty-three gene signature in the TCGA cohort; (C-E) A heatmap of mRNA expression of the twenty-three gene signature, and Risk cures in the TCGA cohort.

A
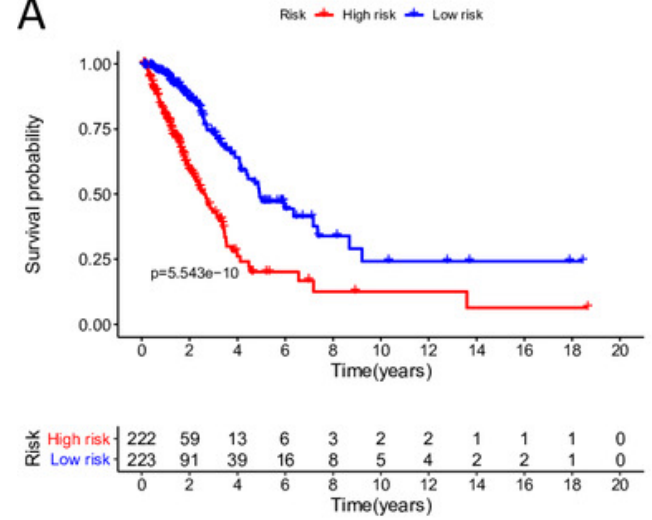

B

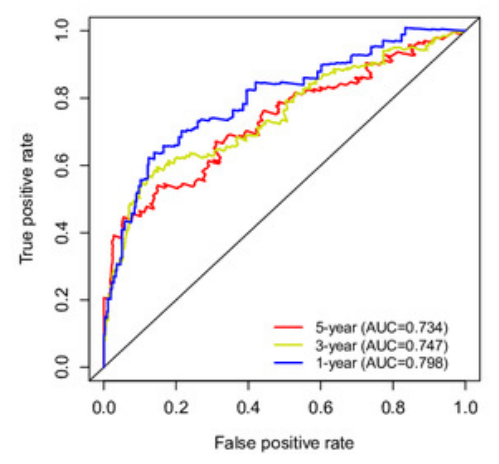

C

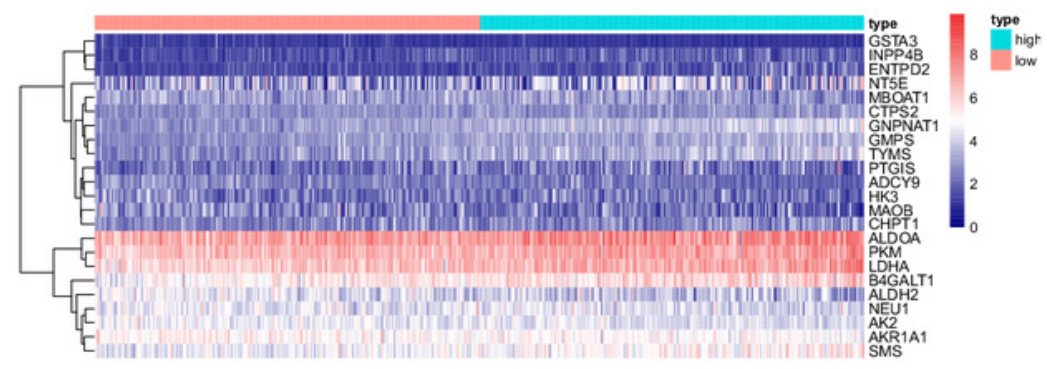

D

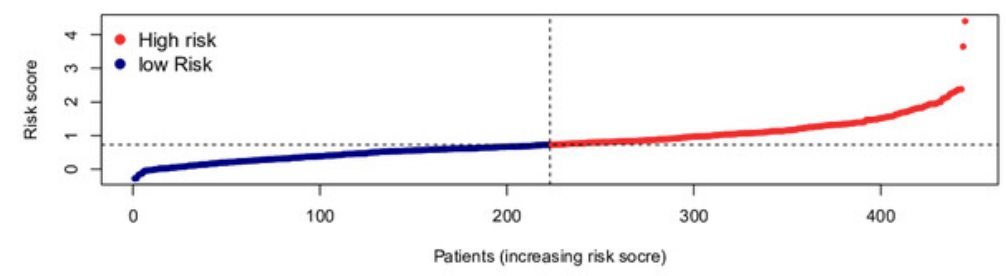

E

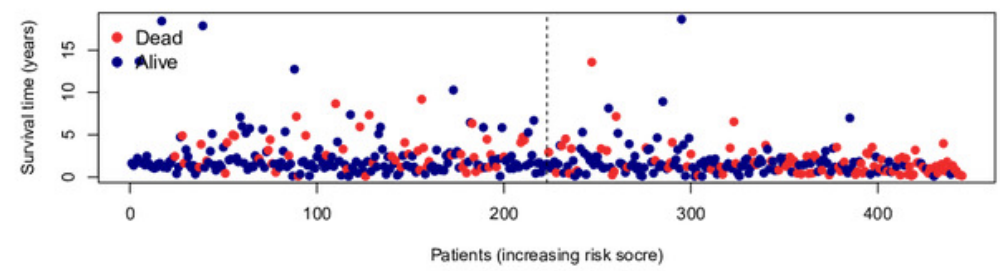


Figure 5

Kaplan-Meier analysis, time-dependent ROC analysis, and risk score analysis for the twenty-three gene signature in the GSE30219 cohort.

(A) Kaplan-Meier curve of the twenty-three gene signature in the GSE30219 cohort; (B) Timedependent ROC analysis of the twenty-three gene signature in the GSE30219 cohort; (C-E) A heatmap of mRNA expression of the twenty-three gene signature, and Risk cures in the GSE30219 cohort.

A

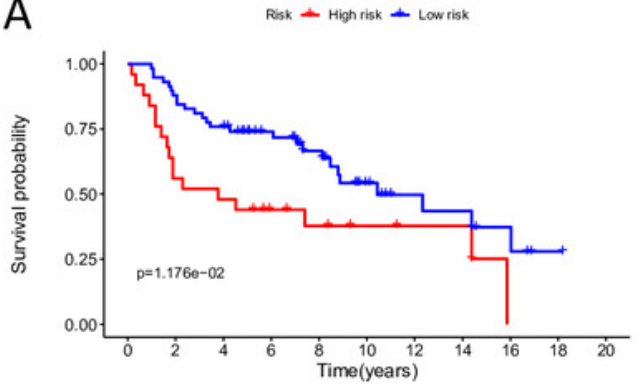

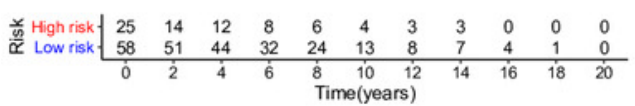

B

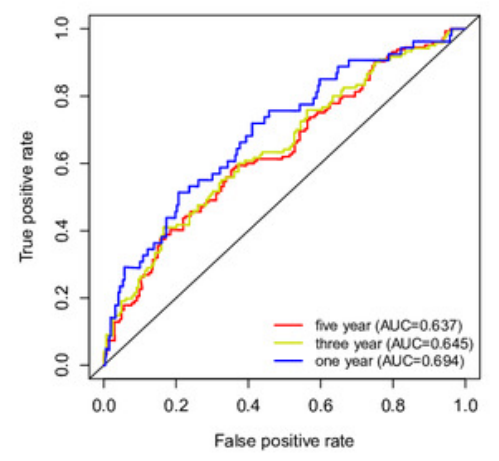

C

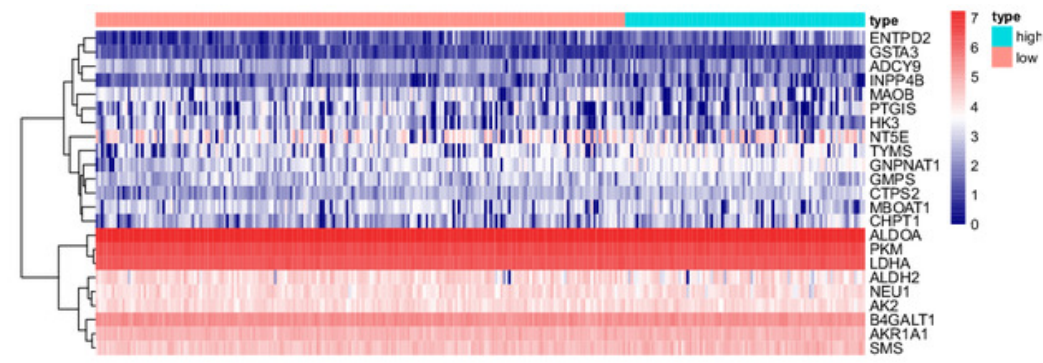

D

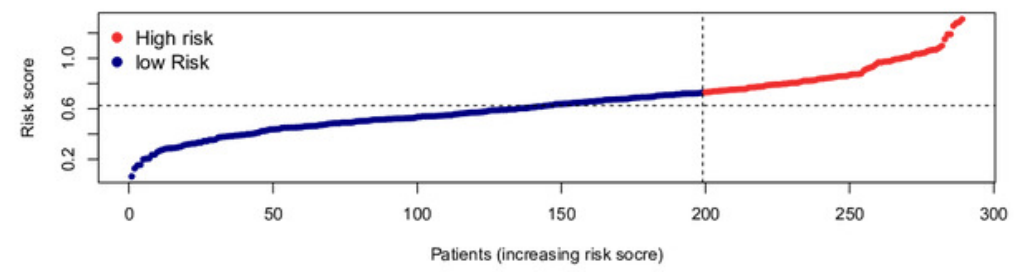

E

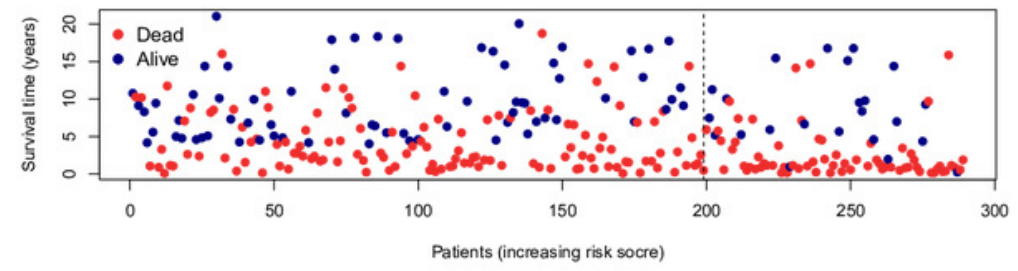




\section{Figure 6}

Kaplan-Meier analysis, time-dependent ROC analysis, and risk score analysis for the twenty-three gene signature in the GSE72094 cohort.

(A) Kaplan-Meier curve of the twenty-three gene signature in the GSE72094 cohort; (B) Timedependent ROC analysis of the twenty-three gene signature in the GSE72094 cohort; (C-E) A heatmap of mRNA expression of the twenty-three gene signature, and Risk cures in the GSE72094 cohort.

A
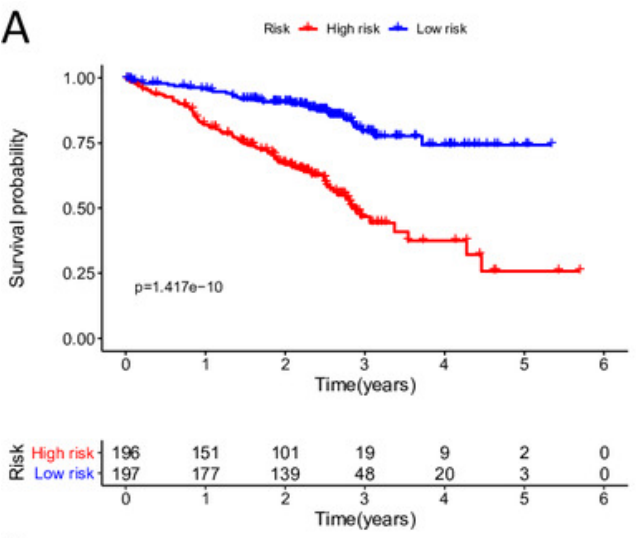

B

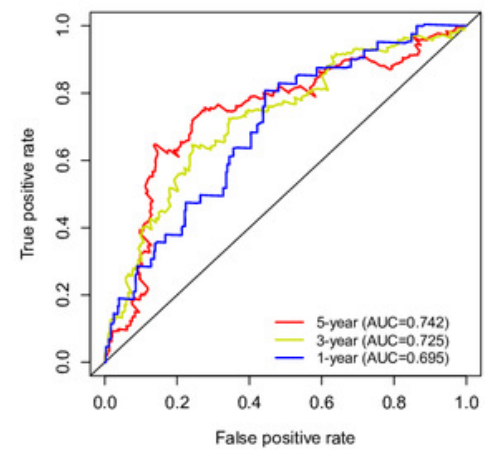

C

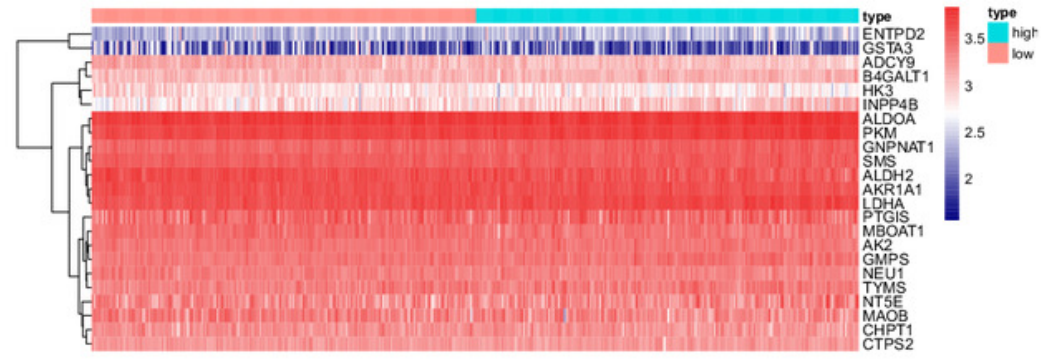

D

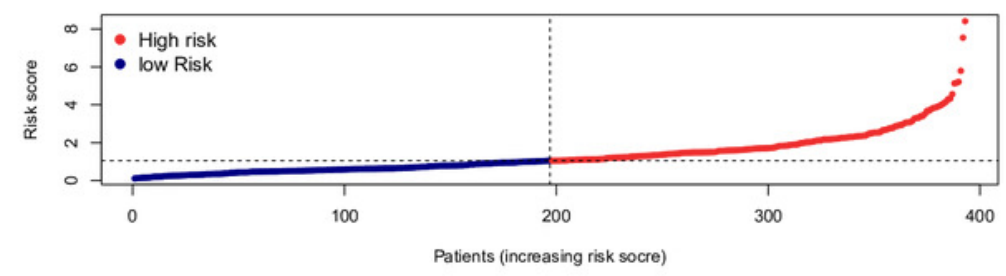

$\mathrm{E}$

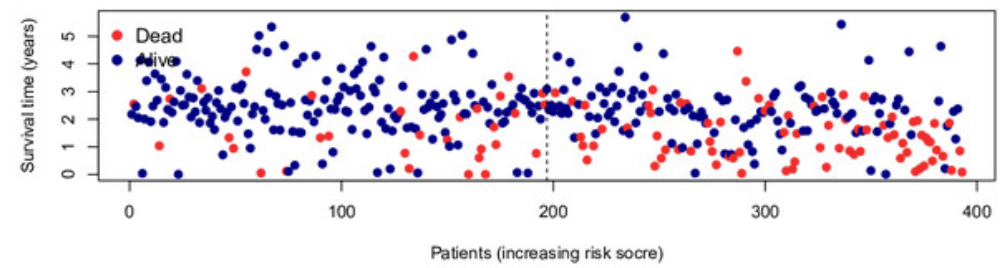




\section{Figure 7}

Forrest plot of the univariate (yellow) and multivariate (red) Cox regression analysis in the each cohort.

(A-B) Forrest plot of the univariate (yellow) and multivariate (red) Cox regression analysis in the TCGA-LUAD cohort; (C-D) Forrest plot of the univariate (yellow) and multivariate (red) Cox regression analysis in the GSE30219 cohort; (E-F) Forrest plot of the univariate (yellow) and multivariate (red) Cox regression analysis in the GSE72094 cohort. 


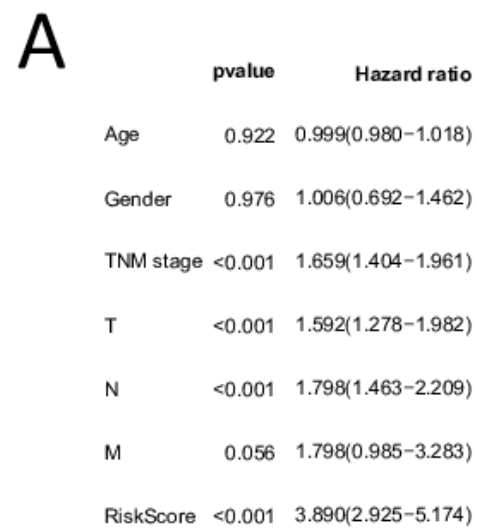

C
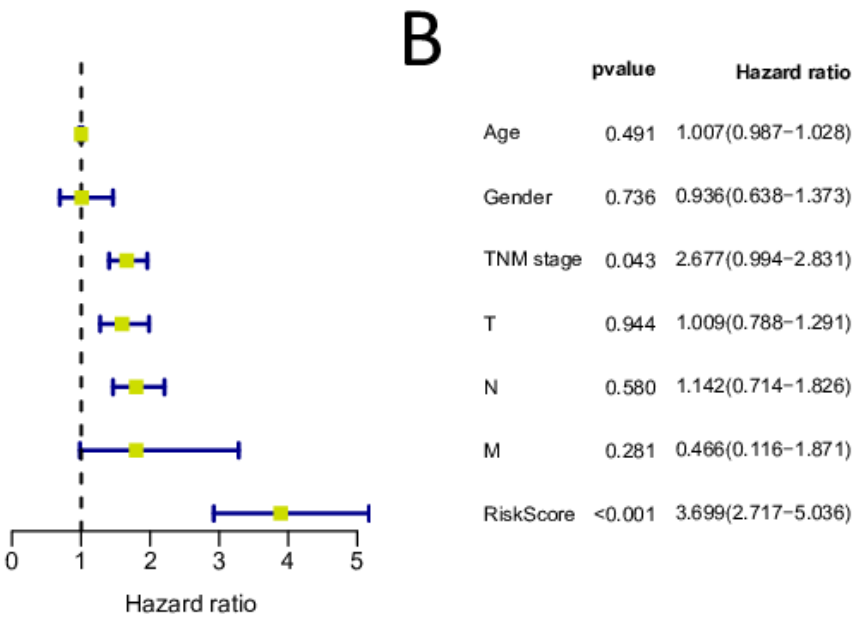

D

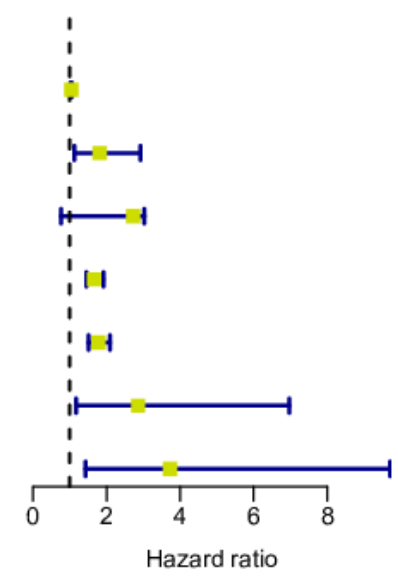

Gender $\quad 0.014 \quad 1.815(1.129-2.917)$

TNM stage $\quad 0.002 \quad 2.719(0.762-3.030)$

$\mathrm{T} \quad<0.001 \quad 1.663(1.448-1.911)$

$\mathrm{N} \quad<0.001 \quad 1.777(1.510-2.091)$

M $\quad 0.021 \quad 2.856(1.170-6.970)$

RiskScore $\quad 0.007 \quad 3.726(1.429-9.714)$

E
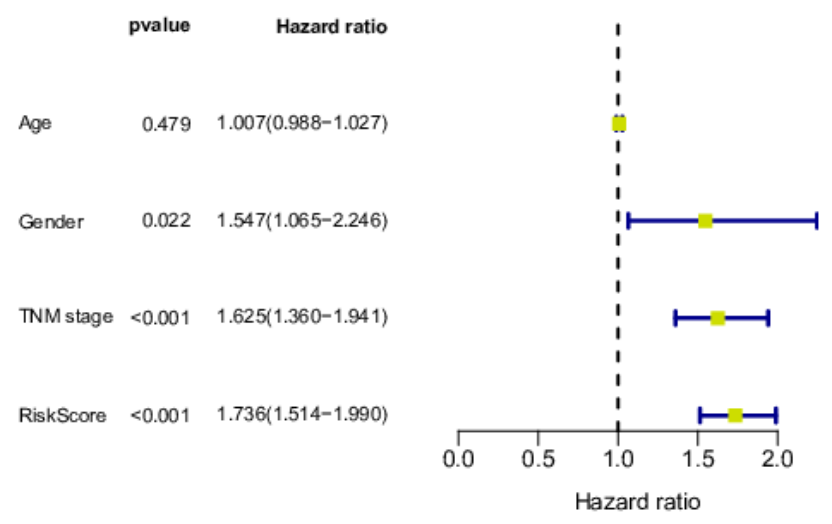

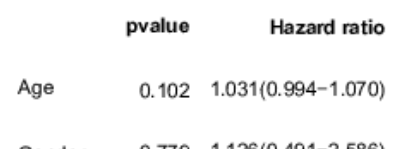

Gender $\quad 0.779 \quad 1.126(0.491-2.586)$

TNM stage $\quad 0.044 \quad 2.409(1.014-2.882)$

$\begin{array}{lll}\mathrm{T} & 0.001 & 1.361(1.126-1.644)\end{array}$

N $\quad 0.007 \quad 1.358(1.087-1.698)$

M $\quad 0.062 \quad 2.357(0.958-5.797)$

RiskScore $\quad 0.016 \quad 2.260(1.168-4.374)$

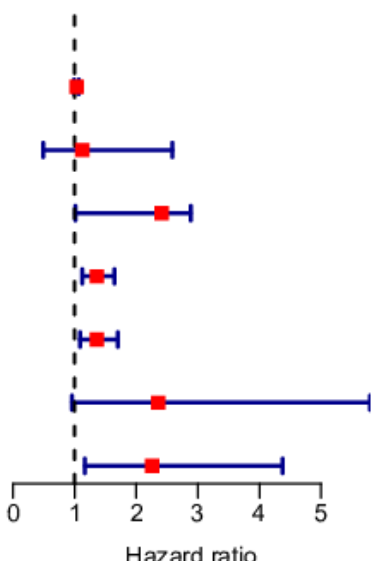

$\mathrm{F}$

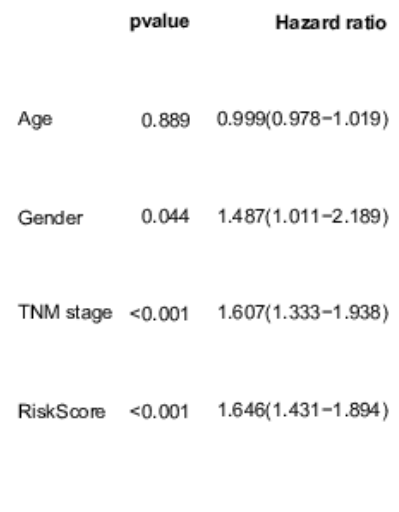




\section{Figure 8}

The Kaplan-Meier curve for the twenty-three gene signature in LUAD.

(A-B) The Kaplan-Meier curve showed that the survival of patients was significantly poorer in the high-risk group in TNM stages I and IITNM stages III and IV of the TCGA-LUAD cohort; (CD) The Kaplan-Meier curve showed that the survival of patients was significantly poorer in the high-risk group in TNM stages I and II/TNM stages III and IV of the GSE30219 cohort. However, there was no statistical significance in TNM stages III and IV; (E-F) The Kaplan-Meier curve showed that the survival of patients was significantly poorer in the highrisk group in TNM stages I and II/TNM stages III and IV of the GSE72094 cohort. 
A Stage I+II Risk - High risk - Low risk

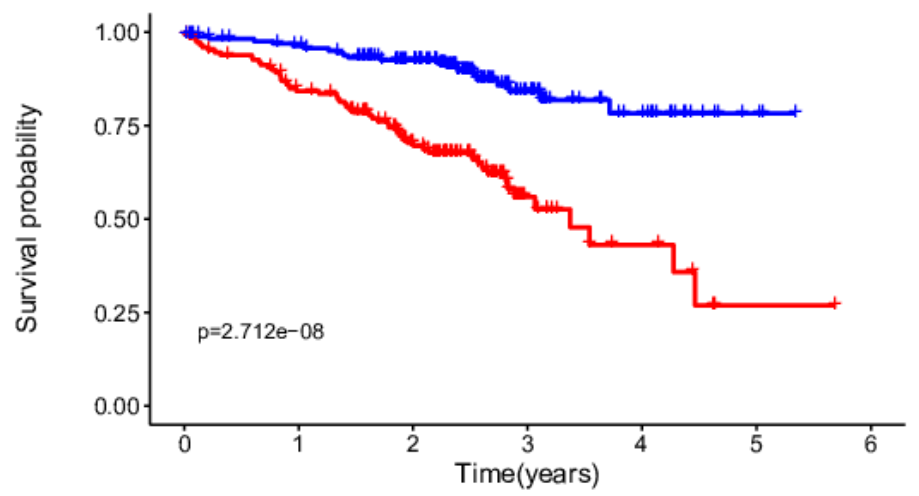

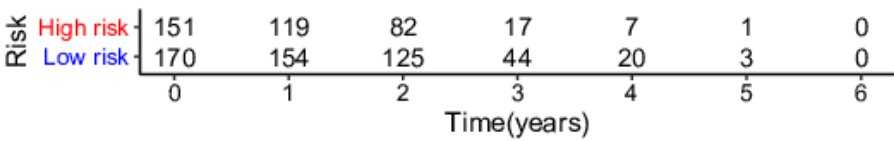

C Stage I+II Risk $\rightarrow$ High risk $\rightarrow$ Low risk

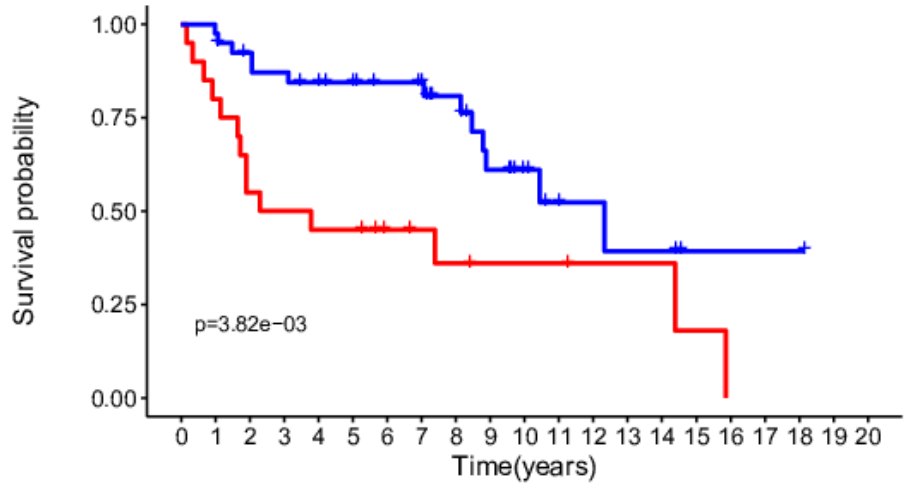

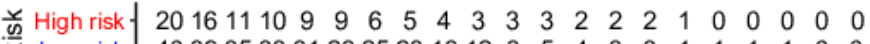

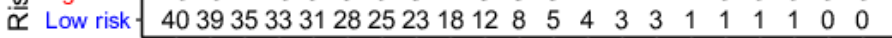
$\begin{array}{lllllllllllllllllllll}0 & 1 & 2 & 3 & 4 & 5 & 6 & 7 & 8 & 9 & 10 & 11 & 12 & 13 & 14 & 15 & 16 & 17 & 18 & 19 & 1 \\ 0\end{array}$ Time(years)

E Stage I+II Risk - High risk + Low risk

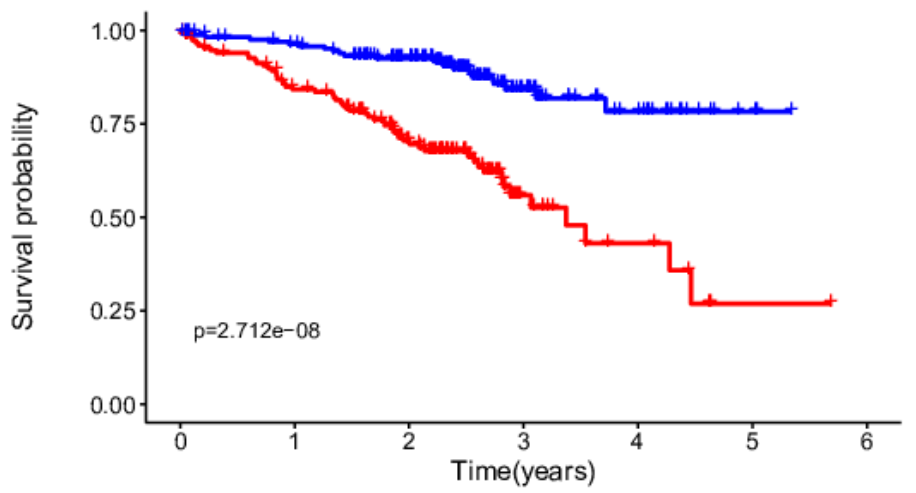

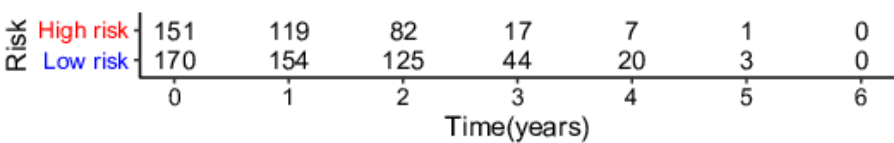

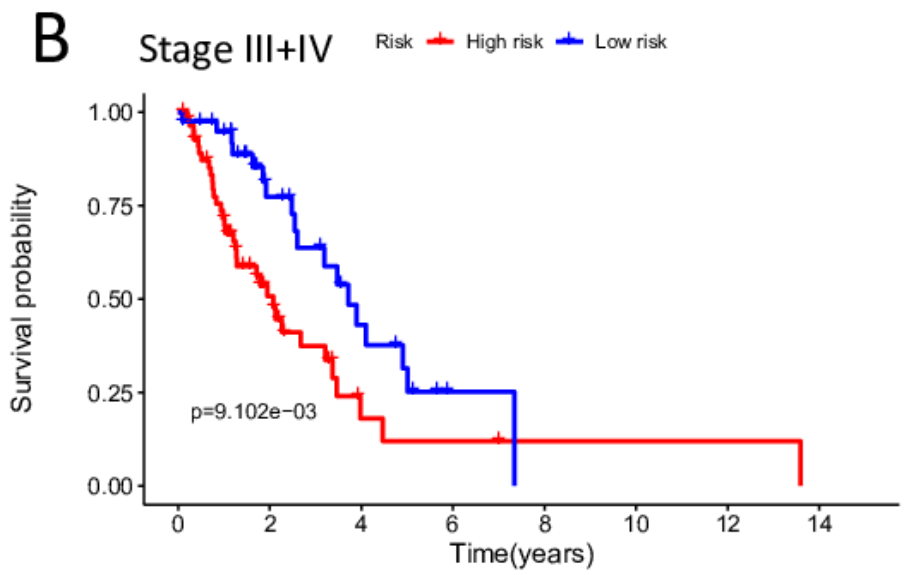

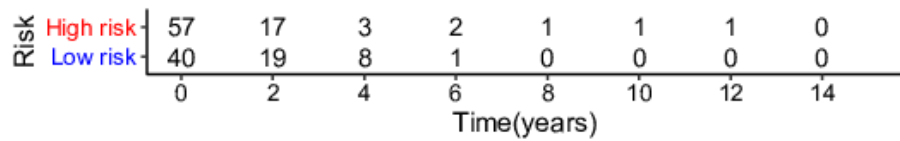

D Stage III+IV Risk - High risk - Low risk

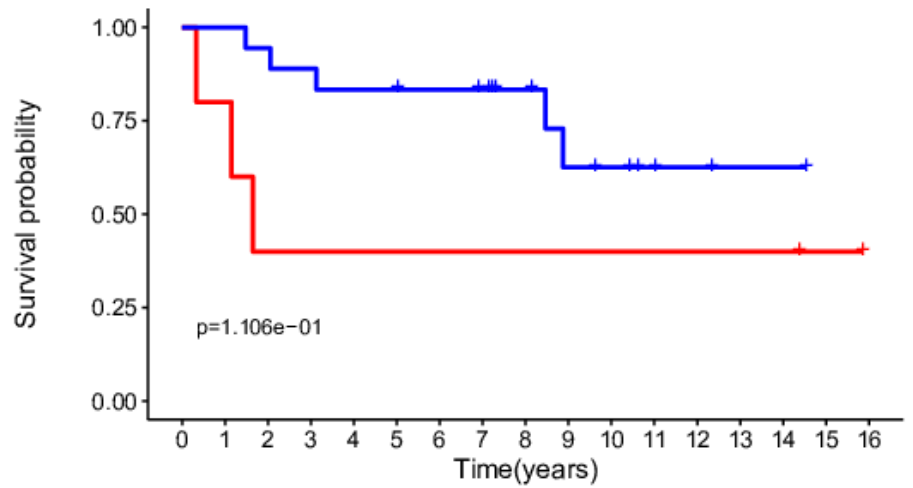

\begin{tabular}{l|lllllllllllllllll} 
w High risk- & 5 & 4 & 2 & 2 & 2 & 2 & 2 & 2 & 2 & 2 & 2 & 2 & 2 & 2 & 2 & 1 & 0
\end{tabular}

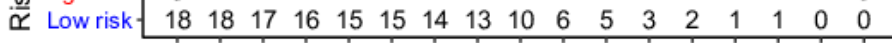
F Stage III+IV Risk + High risk + Low risk

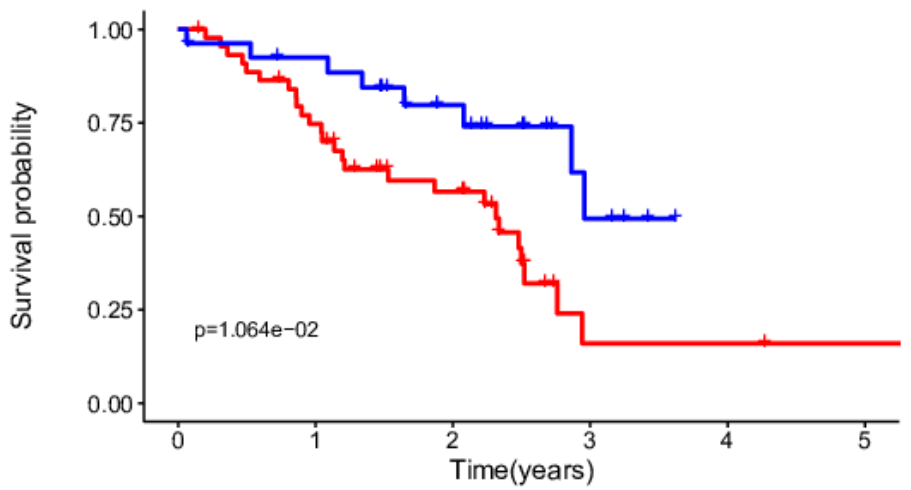

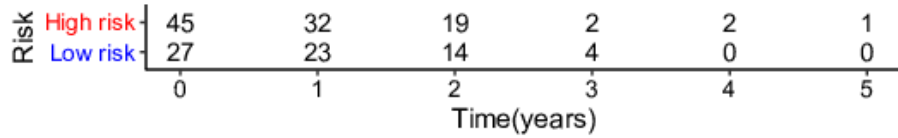


Figure 9

Building the nomogram for predicting the overall survival of LUAD patients from the TCGA cohort.

(A) The nomogram plot was built based on all prognostic factors in the TCGA-LUAD; (B) The calibration plot for internal validation of the nomogram; (C-E) The time-dependent ROC curves of the nomograms compared for 1-, 3-, and 5-year overall survival in TCGA-LUAD.

A

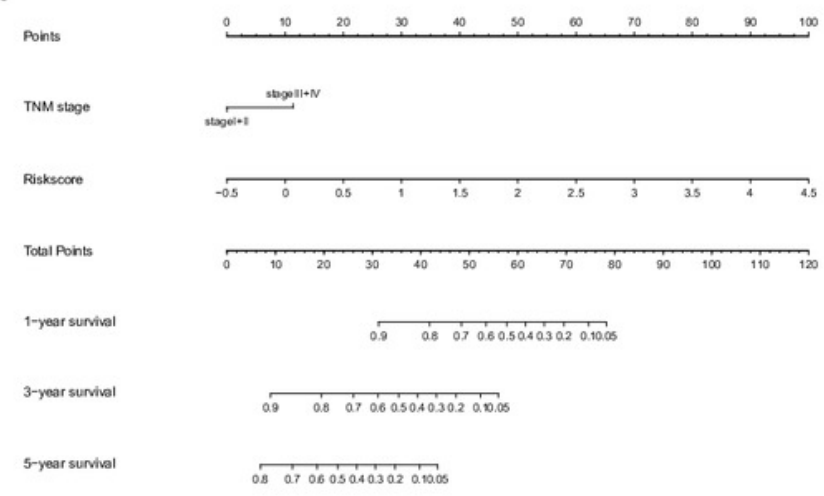

C

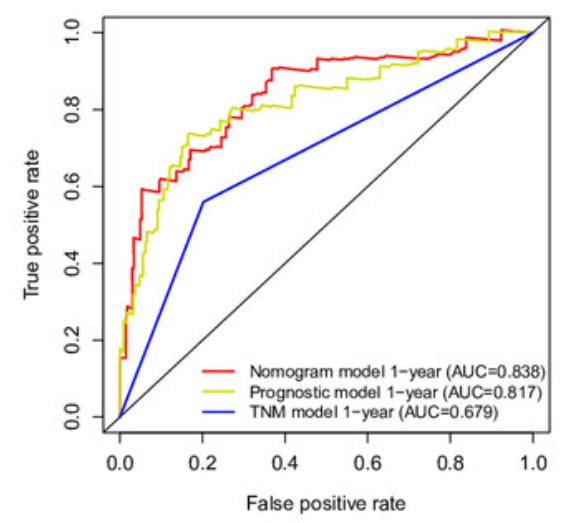

D
B
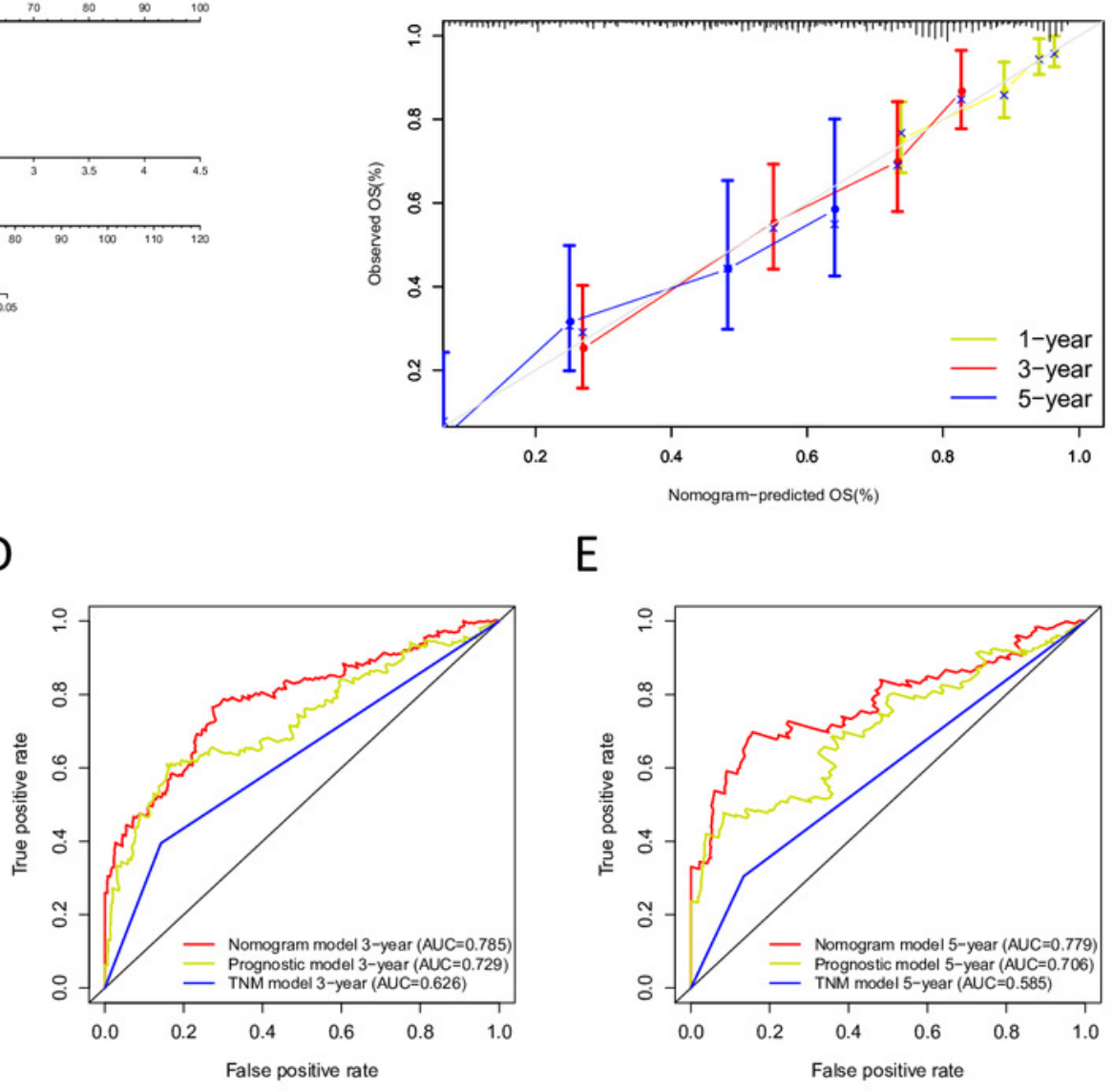

$\mathrm{E}$

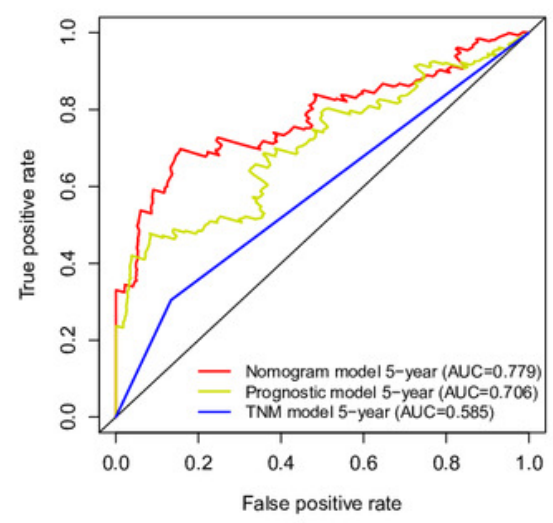


Figure 10

Validation of the nomogram in the GSE30219 and GSE72094 cohorts.

(A) The calibration plot for validation of the nomogram in the GSE30219 cohort; (B-D) the time-dependent ROC curves of the nomograms compared for 1-, 3-, and 5-year overall survival in the GSE30219 cohort; (E) The calibration plot for validation of the nomogram in the GSE72094 cohort; (F-H) The time-dependent ROC curves of the nomograms compared for 1-, 3-, and 5-year overall survival in GSE30219 cohort.

A

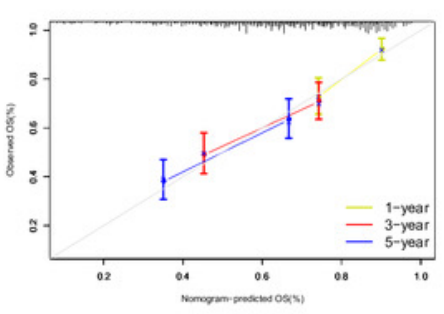

$\mathrm{E}$

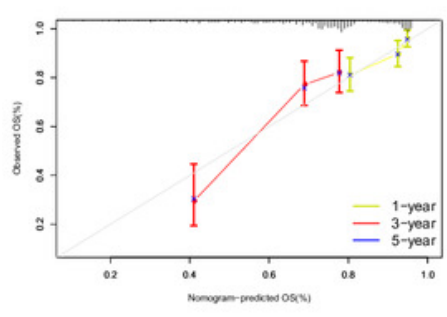

B

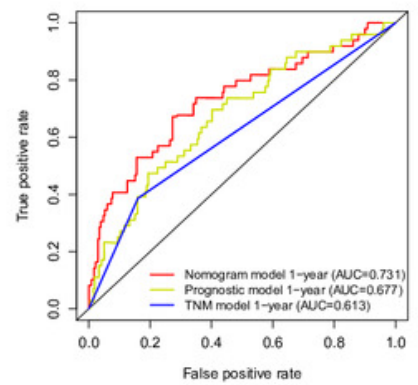

F

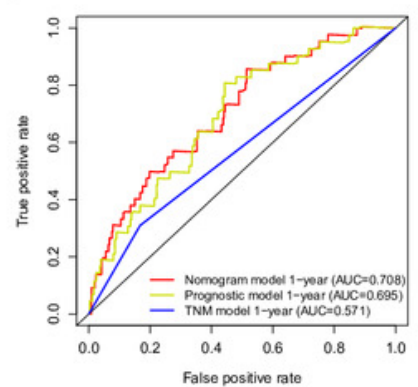

C

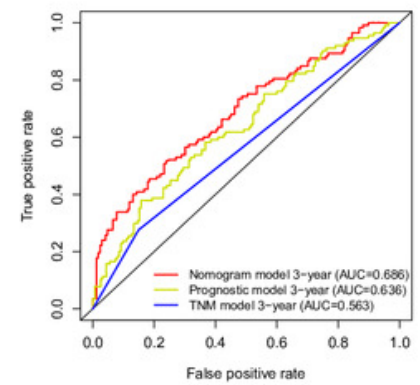

G

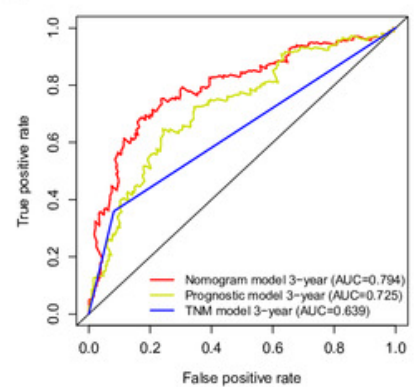

D

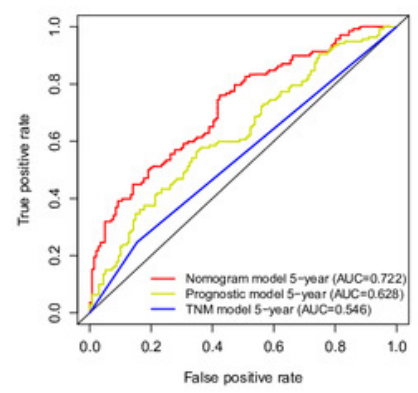

$\mathrm{H}$

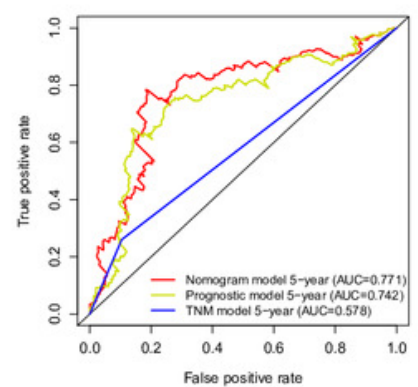


Figure 11

KEGG and GO enrichment pathway analysis.

(A): KEGG enrichment pathway analysis of twenty-three metabolic-related genes obtained by the lasso Cox model: Five representative KEGG pathways in high-risk patients. Five representative KEGG pathways in low-risk patients. (B): GO enrichment pathway analysis of twenty-three metabolic-related genes obtained by the lasso cox model.

A

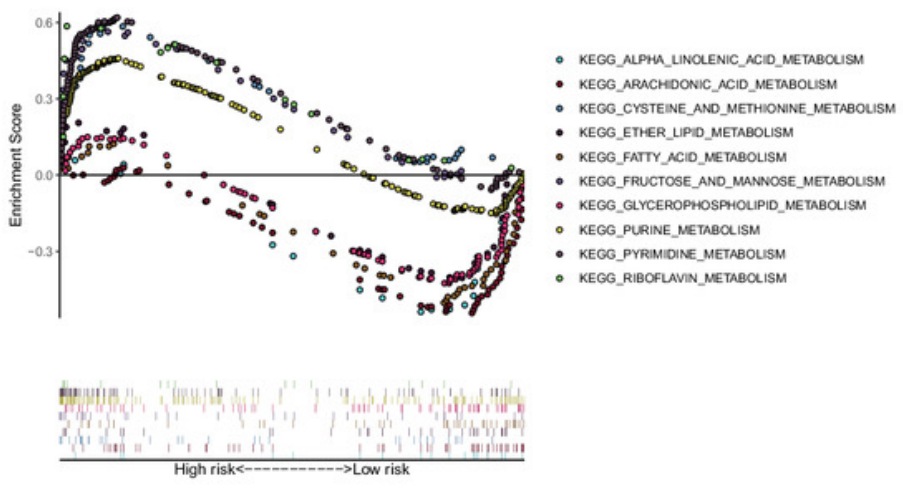

B

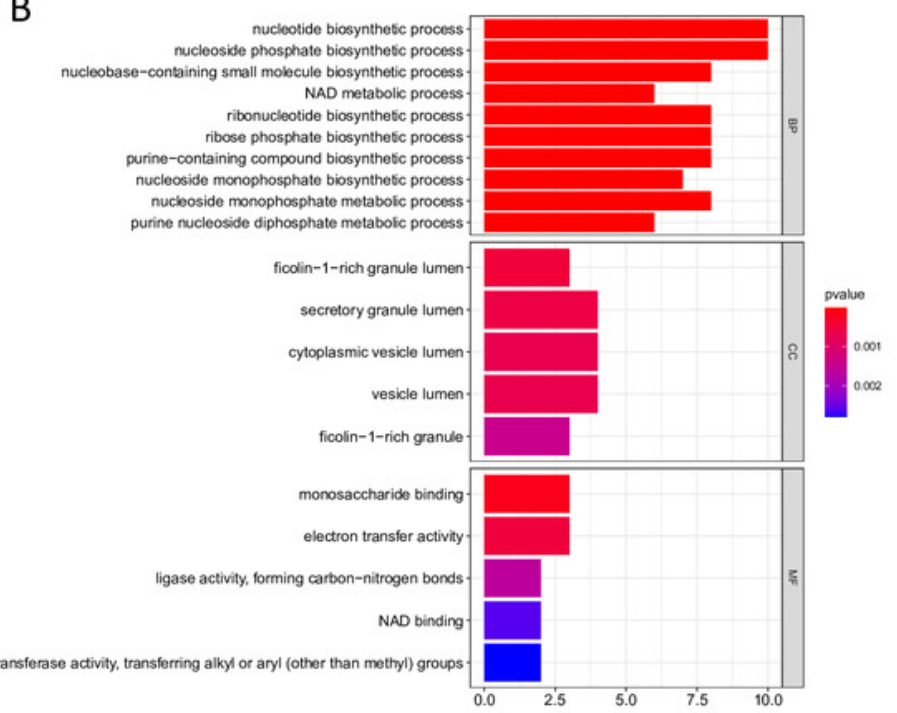




\section{Table $\mathbf{1}$ (on next page)}

The 23-gene signatures screened by their coefficients. 


\begin{tabular}{|c|c|c|c|c|c|}
\hline Gene & Coefficient & HR & HR.95L & HR.95H & $P$ \\
\hline AKR1A1 & -0.005 & 0.985 & 0.973 & 0.996 & 0.01 \\
\hline NT5E & 0.003 & 1.01 & 1.003 & 1.017 & 0.007 \\
\hline PTGIS & 0.01 & 1.007 & 1.003 & 1.011 & 0.001 \\
\hline GMPS & 0.003 & 1.075 & 1.039 & 1.113 & 4.17E-05 \\
\hline MBOAT1 & -0.025 & 0.956 & 0.92 & 0.994 & 0.023 \\
\hline ADCY9 & -0.009 & 0.908 & 0.847 & 0.975 & 0.008 \\
\hline B4GALT1 & 0.002 & 1.011 & 1.006 & 1.016 & $3.05 \mathrm{E}-06$ \\
\hline MAOB & -0.019 & 0.968 & 0.938 & 0.998 & 0.039 \\
\hline INPP4B & 0.046 & 1.122 & 1.033 & 1.219 & 0.006 \\
\hline NEU1 & -0.003 & 0.986 & 0.972 & 0.999 & 0.034 \\
\hline ALDOA & 0.001 & 1.002 & 1 & 1.003 & 0.006 \\
\hline ENTPD2 & 0.065 & 1.119 & 1.062 & 1.179 & $2.25 \mathrm{E}-05$ \\
\hline GNPNAT1 & 0.028 & 1.045 & 1.029 & 1.061 & $2.86 \mathrm{E}-08$ \\
\hline GSTA3 & -0.084 & 0.708 & 0.502 & 0.997 & 0.048 \\
\hline PKM & 0.001 & 1.004 & 1.002 & 1.007 & 0 \\
\hline HK3 & -0.008 & 0.946 & 0.904 & 0.99 & 0.016 \\
\hline ALDH2 & -0.001 & 0.98 & 0.966 & 0.993 & 0.003 \\
\hline AK2 & -0.013 & 0.978 & 0.96 & 0.995 & 0.014 \\
\hline LDHA & 0.002 & 1.005 & 1.003 & 1.006 & $4.20 \mathrm{E}-09$ \\
\hline CHPT1 & 0.024 & 1.042 & 1.008 & 1.076 & 0.014 \\
\hline SMS & 0.001 & 1.013 & 1.007 & 1.02 & 0 \\
\hline CTPS2 & 0.052 & 1.079 & 1.016 & 1.146 & 0.014 \\
\hline TYMS & 0.016 & 1.028 & 1.014 & 1.044 & 0.0002 \\
\hline
\end{tabular}

HR: hazard ratio. 
Table 2 (on next page)

Comparison of the twenty-three gene prognostic signature to the two published prognostic signatures. 


\section{PeerJ}

\begin{tabular}{|c|c|c|c|c|c|c|c|c|c|}
\hline \multirow{2}{*}{ Studies } & \multicolumn{4}{|c|}{ TCGA cohort } & \multicolumn{2}{|c|}{ GSE30219 cohort } & \multicolumn{3}{|c|}{ GSE72094 cohort } \\
\hline & HR $(95 \% \mathrm{CI})$ & $P$ & C-index & HR $(95 \% \mathrm{CI})$ & $P$ & C-index & HR $(95 \% \mathrm{CI})$ & $P$ & C-index \\
\hline Present study & $4.55(3.487 \sim 5.946)$ & $2.00 \mathrm{E}-16$ & 0.730 & $3.80(1.977 \sim 7.308)$ & $6.25 \mathrm{E}-05$ & 0.682 & $1.74(1.514 \sim 1.99)$ & $2.96 \mathrm{E}-15$ & 0.709 \\
\hline \multicolumn{10}{|l|}{ 23-gene signature } \\
\hline Zhao, K.et al & $1.10(1.073 \sim 1.123)$ & $6.20 \mathrm{E}-16$ & 0.697 & $1.98(1.683 \sim 2.319)$ & $1.72 \mathrm{E}-19$ & 0.682 & $1.53(1.39 \sim 1.672)$ & $2.17 \mathrm{E}-15$ & 0.704 \\
\hline \multicolumn{10}{|l|}{ 20-gene signature } \\
\hline $\begin{array}{c}\text { Xie, H.et al } \\
\text { 6-gene signature }\end{array}$ & $1.29(1.167 \sim 1.428)$ & 7.45E-07 & 0.632 & $2.34(1.825 \sim 2.996)$ & $1.87 \mathrm{E}-11$ & 0.668 & $1.82(1.506 \sim 2.206)$ & $7.10 \mathrm{E}-10$ & 0.660 \\
\hline
\end{tabular}

HR: hazard ratio; CI: confidence interval. 


\section{Table 3 (on next page)}

Correlation analysis of the clinical characteristics and the twenty-three gene signature in LUAD. 


\begin{tabular}{|c|c|c|c|c|c|c|c|c|c|c|c|c|c|c|c|}
\hline \multirow{2}{*}{ Characteristics } & \multicolumn{5}{|c|}{ TCGA training cohort(n=445) } & \multicolumn{5}{|c|}{ GSE30219 testing cohort(n=83) } & \multicolumn{5}{|c|}{ GSE72094 testing cohort(n=393) } \\
\hline & $\mathrm{n}$ & Mean (rick score) & SD & $\mathrm{t}$ & $P$ & $\mathrm{n}$ & Mean (rick score) & SD & $\mathrm{t}$ & $P$ & $\mathrm{n}$ & Mean (rick score) & SD & $\mathrm{t}$ & $P$ \\
\hline \multicolumn{16}{|l|}{ Age(years) } \\
\hline$<68$ & 247 & 0.765 & 0.64 & -0.164 & 0.87 & 23 & 0.543 & 0.346 & -0.088 & 0.931 & 153 & 1.253 & 0.93 & -0.524 & 0.600 \\
\hline$\geq 68$ & 198 & 0.775 & 0.663 & & & 60 & 0.55 & 0.272 & & & 240 & 1.305 & 1.04 & & \\
\hline \multicolumn{16}{|l|}{ Gender } \\
\hline Female & 244 & 0.702 & 0.611 & -2.393 & 0.017 & 19 & 0.535 & 0.357 & -0.183 & 0.856 & 219 & 1.203 & 1.012 & -1.850 & 0.065 \\
\hline Male & 201 & 0.851 & 0.686 & & & 64 & 0.552 & 0.274 & & & 174 & 1.389 & 0.972 & & \\
\hline \multicolumn{16}{|l|}{ TNM stage } \\
\hline Stage I+II & 348 & 0.68 & 0.584 & -4.822 & $<0.001$ & 60 & 0.456 & 0.235 & -6.598 & $<0.001$ & 320 & 1.166 & 0.842 & -3.771 & $<0.001$ \\
\hline Stage III+IV & 97 & 1.083 & 0.765 & & & 23 & 0.875 & 0.239 & & & 73 & 1.805 & 1.392 & & \\
\hline \multicolumn{16}{|l|}{$\mathrm{T}$} \\
\hline $\mathrm{T} 1+2$ & 387 & 0.716 & 0.595 & -3.5 & 0.001 & 73 & 0.528 & 0.299 & -2.479 & 0.024 & - & - & - & - & - \\
\hline $\mathrm{T} 3+4$ & 58 & 1.125 & 0.86 & & & 10 & 0.697 & 0.185 & & & - & - & - & & \\
\hline \multicolumn{16}{|l|}{$\mathrm{N}$} \\
\hline No & 292 & 0.71 & 0.639 & -2.629 & 0.009 & 71 & 0.502 & 0.276 & -4.03 & 0.001 & - & - & - & - & - \\
\hline $\mathrm{N} 1-3$ & 153 & 0.881 & 0.657 & & & 12 & 0.817 & 0.245 & & & - & - & - & & \\
\hline \multicolumn{16}{|l|}{ M } \\
\hline M0 & 403 & 0.742 & 0.64 & -2.577 & 0.013 & 67 & 0.468 & 0.241 & -5.959 & $<0.001$ & - & - & - & - & - \\
\hline M1 & 42 & 1.028 & 0.689 & & & 16 & 0.881 & 0.251 & & & - & - & - & & \\
\hline
\end{tabular}

1 SD: standard deviation; TNM: tumor-node metastasis. 


\section{Table 4 (on next page)}

Univariate and multivariate cox regression analysis of overall survival in each cohort. 


\begin{tabular}{|c|c|c|c|c|c|c|c|c|}
\hline \multirow{2}{*}{ Variables } & \multicolumn{4}{|c|}{ Univariate analysis } & \multicolumn{4}{|c|}{ Multivariate analysis } \\
\hline & HR & HR.95L & HR.95H & $P$ & $\mathrm{HR}$ & HR.95L & HR.95H & $P$ \\
\hline \multicolumn{9}{|c|}{ TCGA training cohort $(\mathrm{n}=445)$} \\
\hline $\operatorname{Age}(\geq 68 /<68)$ & 0.999 & 0.98 & 1.018 & 0.922 & 1.007 & 0.987 & 1.028 & 0.491 \\
\hline Gender(female/male) & 1.006 & 0.692 & 1.462 & 0.976 & 0.936 & 0.638 & 1.373 & 0.736 \\
\hline TNM stage $(\mathrm{I}+\mathrm{II} / \mathrm{III}+\mathrm{IV})$ & 1.659 & 1.404 & 1.961 & 3.01E-09 & 2.677 & 0.994 & 2.831 & 0.043 \\
\hline $\mathrm{T}$ & 1.592 & 1.278 & 1.982 & $3.27 \mathrm{E}-05$ & 1.009 & 0.788 & 1.291 & 0.944 \\
\hline $\mathrm{N}$ & 1.798 & 1.463 & 2.209 & $2.34 \mathrm{E}-08$ & 1.142 & 0.714 & 1.826 & 0.58 \\
\hline M & 1.798 & 0.985 & 3.283 & 0.056 & 0.466 & 0.116 & 1.871 & 0.281 \\
\hline Risk Score & 3.89 & 2.925 & 5.174 & $9.79 \mathrm{E}-21$ & 3.699 & 2.717 & 5.036 & $9.80 \mathrm{E}-17$ \\
\hline \multicolumn{9}{|c|}{ GSE30219 testing cohort(n=83) } \\
\hline $\operatorname{Age}(\geq 68 /<68)$ & 1.037 & 1.023 & 1.052 & $3.22 \mathrm{E}-07$ & 1.031 & 0.994 & 1.07 & 0.102 \\
\hline Gender(female/male) & 1.815 & 1.129 & 2.917 & 0.014 & 1.126 & 0.491 & 2.586 & 0.779 \\
\hline TNM stage (I+II/III+IV) & 2.719 & 0.762 & 3.03 & $2.35 \mathrm{E}-03$ & 2.409 & 1.014 & 2.882 & 0.044 \\
\hline $\mathrm{T}$ & 1.663 & 1.448 & 1.911 & $7.10 \mathrm{E}-13$ & 1.361 & 1.126 & 1.644 & 0.001 \\
\hline $\mathrm{N}$ & 1.777 & 1.51 & 2.091 & $4.36 \mathrm{E}-12$ & 1.358 & 1.087 & 1.698 & 0.007 \\
\hline M & 2.856 & 1.17 & 6.97 & 0.021 & 2.357 & 0.958 & 5.797 & 0.062 \\
\hline Risk Score & 3.726 & 1.429 & 9.714 & 0.007 & 2.26 & 1.168 & 4.374 & 0.016 \\
\hline \multicolumn{9}{|c|}{ GSE72094 testing cohort( $\mathrm{n}=393)$} \\
\hline $\operatorname{Age}(\geq 68 /<68)$ & 1.007 & 0.988 & 1.027 & 0.479 & 0.999 & 0.978 & 1.019 & 0.889 \\
\hline Gender(female/male) & 1.547 & 1.065 & 2.246 & 0.022 & 1.487 & 1.011 & 2.189 & 0.044 \\
\hline TNM stage & 1.625 & 1.360 & 1.941 & $<0.001$ & 1.607 & 1.333 & 1.938 & $<0.001$ \\
\hline Risk Score & 1.736 & 1.514 & 1.990 & $<0.001$ & 1.646 & 1.431 & 1.894 & $<0.001$ \\
\hline
\end{tabular}

HR: hazard ratio; TNM: tumor-node metastasis. 


\section{Table 5 (on next page)}

Comparison of the nomogram model with the TNM model and prognostic model. 


\begin{tabular}{|c|c|c|c|c|c|c|c|c|c|}
\hline \multirow{2}{*}{ Cohort } & \multicolumn{3}{|c|}{ Nomogram model } & \multicolumn{3}{|c|}{ TNM model } & \multicolumn{3}{|c|}{ Prognostic model } \\
\hline & HR $(95 \% \mathrm{CI})$ & $P$ & C-index & $\mathrm{HR}(95 \% \mathrm{CI})$ & $P$ & C-index & $\mathrm{HR}(95 \% \mathrm{CI})$ & $P$ & C-index \\
\hline TCGA cohort & $2.719(2.269 \sim 3.259)$ & $2.00 \mathrm{E}-16$ & 0.793 & $2.957(2.015 \sim 4.339)$ & $3.03 \mathrm{E}-08$ & 0.654 & $4.55(3.487 \sim 5.946)$ & $2.00 \mathrm{E}-16$ & 0.730 \\
\hline GSE30219 cohort & $2.958(2.189 \sim 3.375)$ & $7.55 \mathrm{E}-16$ & 0.684 & $1.267(0.889 \sim 1.803)$ & 0.016 & 0.619 & $3.80(1.977 \sim 7.308)$ & $6.25 \mathrm{E}-05$ & 0.682 \\
\hline GSE72094 cohort & $2.718(2.181 \sim 3.388)$ & $7.55 \mathrm{E}-16$ & 0.713 & $2.606(1.736 \sim 3.914)$ & $3.85 \mathrm{E}-06$ & 0.579 & $1.74(1.514 \sim 1.99)$ & $2.96 \mathrm{E}-15$ & 0.709 \\
\hline
\end{tabular}

TNM: tumor-node metastasis; CI: confidence interval. 
Table 6(on next page)

The SIZE, ES, NES. NOM $p$-val, and FDR q-val of the KEGG enrichment pathways. 


\begin{tabular}{|c|c|c|c|c|c|}
\hline & SIZE & ES & NES & NOM $P$ & FDR q-valve \\
\hline \multicolumn{6}{|l|}{ Low risk group enrich pathways } \\
\hline KEGG_ASTHMA & 28 & -0.802 & -2.039 & $<0.01$ & 0.040 \\
\hline KEGG_ARACHIDONIC_ACID_METABOLISM & 58 & -0.558 & -1.956 & 0.002 & 0.057 \\
\hline KEGG_GLYCEROPHOSPHOLIPID_METABOLISM & 77 & -0.435 & -1.739 & 0.008 & 0.117 \\
\hline KEGG_ALDOSTERONE_REGULATED_SODIUM_REABSORPTION & 42 & -0.494 & -1.702 & 0.010 & 0.135 \\
\hline KEGG_ALPHA_LINOLENIC_ACID_METABOLISM & 19 & -0.568 & -1.672 & 0.019 & 0.136 \\
\hline KEGG_FATTY_ACID_METABOLISM & 42 & -0.517 & -1.635 & 0.048 & 0.138 \\
\hline \multicolumn{6}{|l|}{ High risk group enrich pathways } \\
\hline KEGG_CELL_CYCLE & 124 & 0.746 & 2.384 & $<0.01$ & 0.001 \\
\hline KEGG_PYRIMIDINE_METABOLISM & 98 & 0.620 & 2.160 & $<0.01$ & 0.008 \\
\hline KEGG_MISMATCH_REPAIR & 23 & 0.852 & 2.149 & $<0.01$ & 0.005 \\
\hline KEGG_UBIQUITIN_MEDIATED_PROTEOLYSIS & 134 & 0.541 & 2.144 & $<0.01$ & 0.004 \\
\hline KEGG_P53_SIGNALING_PATHWAY & 68 & 0.546 & 2.100 & 0.002 & 0.006 \\
\hline
\end{tabular}

ES: enrichment score; NES: normalized enrichment score; NOM P: nominal p value; FDR q-valve: false discovery rate. 